QK329

. R6

Rossi, Ljudevit

Floristička istraživanja po jugoistočnoj hrvatskoj 


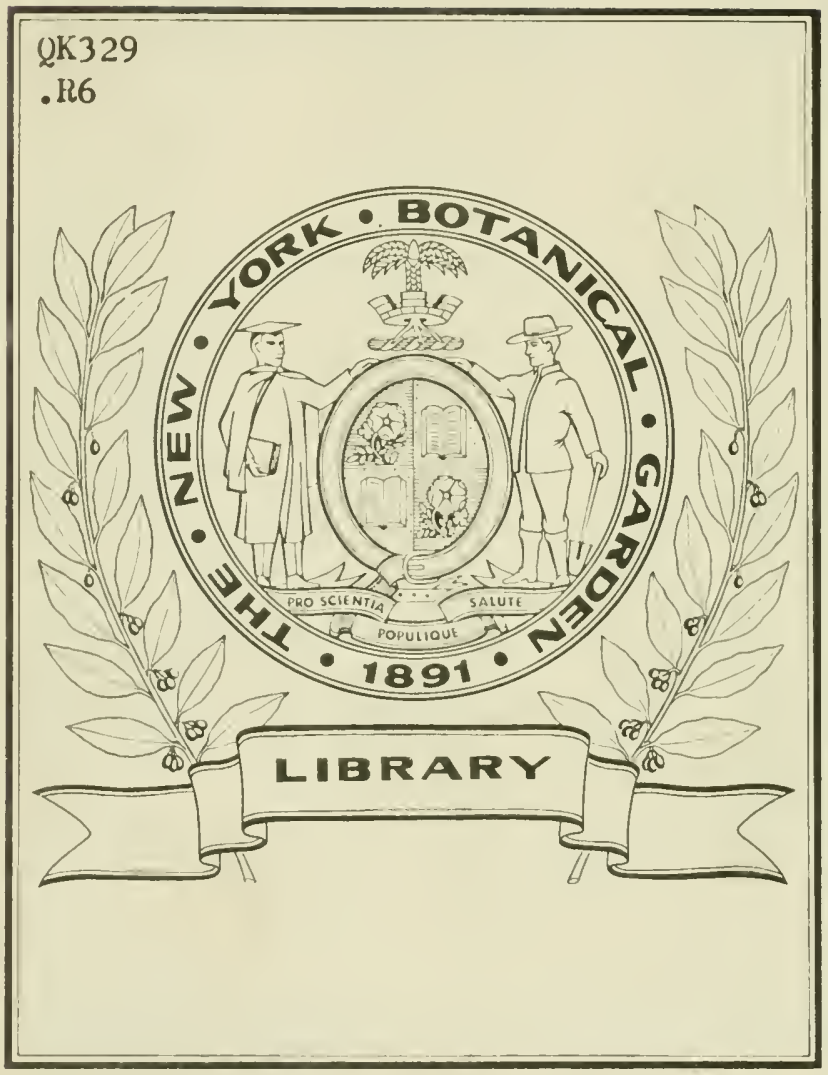





\section{Poklanja pisac.}

\section{FLORISTIČKA ISTRAŽIVANJA}

$\mathrm{PO}$

\section{JUGOISTOČNOJ HRVATSKOJ.}

NAPISAO

LJUDEVIT ROSSI.

POSEBAN OTISAK IZ „GLASNIKA HRVATSKOGA PRIRODOSLOVNOGA DRUṠTVA“ GODIŠTA XXVII. 1915.

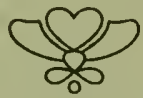

U ZAGREBU 1915.

TISAK KR. ZEMALJSKE TISKARE. 



\section{FLORISTIČKA ISTRAŽIVANJA} po

\section{JUGOISTOČNOJ HRVATSKOJ.}

NAPISAO

\section{LJUDEVIT ROSSI.}

POSEBAN OTISAK IZ „GLASNIKA HRVATSKOGA PRIRODOSLOVNOGA DRUŠTVA“ GODIŠTA XXVII. 1915.

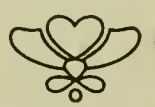

U ZAGREBU 1915. TISAK KR. ZEMALJSKE TISKARE. 



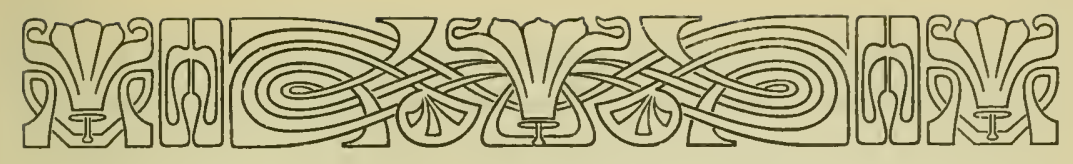

Kad pregledamo Floru hrvatsku bud Neilreichovu ${ }^{1}$ bud Śloser-Vukotinovićevu², opazit ćemo veliku prazninu u navodu bilina iz jugoistočnih krajeva Hrvatske t. j. današnjeg Gračarskog kotara, koji se prostire između južne Plješivice i jugoistočnog Velebita. Prvi nam navodi sa Crnopca 12, Poštaka 7, Ploče 6 a Trvorhe 2, dočim potonji spominju samo sa Crnopca 3 a Ploče 2 vrsti.

Premda je od izdanja ovih Cvjetana proteklo već preko 40 godina, nije se ipak našlo ljudi, koji bi se odlučili, da potanje prouče zanimivu floru ovoga kraja. Uzrok ležat će po svoj prilici u tomu, što su ovi predjeli odveć udaljeni od glavnih prometnih pruga, pa je putovanje stoga skopčano s velikim troškovima i znatnim gubitkom vremena.

Od južno istočnog Velebita bijaše nam dosele poznat samo Crnopac a od južne Plješivice Poštak, koje je oboje pohodio početkom šestdesetih godina prošlog stoljeća kustos Zelebor sabrav na svom zooložkom putovanju mimogredce nekoje biline, koje je Dr. Reichardt ${ }^{3}$ označio i koje su većim dijelom jur poprimljene u Neilreichovoj flori.

U srpnju god. 1978. bijahu Šloser i Vukotinović u Zrmanji, pa je na tom putu ubrane nekoje vrsti izmedu Vrela u Zrmanji i Gračaca naveo sin prvašnjeg Levin u svom putopisu.

Wien 1868.

${ }^{1}$ Neilreich Dr. A., Die Vegetations-Verhältnisse von Croatien.

2 Schlosser Dr. I. C. eques de Klekovski et Farkaš-Vukotinović L. nob., Flora Croatica. Zagrabiae 1869.

${ }^{3}$ Reichardt Dr. H. W. Beitrag zur Flora der Militärgrenze Croatiens. Verh. d. zool. bot. Ges. Wien XVII. p. 765--767.

+ Klekovski L. Putopisne crtice iz gornje Krajine.

Vienac. Zagreb 1881. br. 1, 2, 6-10.

Ljudevit Rossi : Floristička istraživanja po jugoistočnoj Hrvatskoj. 
Iza njih bijaše nakon malne 20 godina $E$. Janchen ${ }^{1}$ na Crnopcu prešavši sutradan Veliku Vrbicu 1180 pravcem Obrovca, nu on nam u svojoj radnji nabraja samo 8 vrsti sa Crnopca.

Dr. A. v. Degen ${ }^{2}$ polıodio je Crnopac, Kitu Velebita i okolicu Gračaca, pa su nekoje njegove nalaze spomenuli $\mathrm{Z}$ a h n ${ }^{3}$, Gáye ${ }^{4}$, Janche $n^{5}$ i $\mathrm{Szab}$ o $^{6}$ dočim će ostale obrete navesti on sám u svojoj Flori velebitskoj.

Osim toga pobilježiše W ald ste in $\mathrm{Kita}$ ibe $\mathrm{l}^{7}$, P o s char$\mathrm{sky}^{8}$ i Dr. B e ck $\mathrm{k}^{9}$ oko Ploče neznatan broj vrsti, papratnjače pako rečenog područja većim su dijelom već objelodanjene ${ }^{10}$.

Vrijedno je jošte istaknuti, da je $\mathrm{Hacquet}{ }^{11}$ bio prvi, koj nam u svom djelu napominje poimence dvije biline $\mathrm{i}$ to: Carlina Velebit.

${ }^{1}$ Janchen E. Eine bot. Reise in die Dinarischen Alpen und den

Mith. d. nat. Ver. an der Univ. Wien VI. p. 69-97.

${ }^{2}$ D egen Dr. A. von. Zwölf neue Pflanzen der Länder der ungar. Krone.

M. bot. lap. Vl. p. 122-129.

${ }^{3} \mathrm{Zahn} \mathrm{K}$. H. Beiträge zur Kenntniss der Hieracien Ungarns und der Balkanländer.

M. bot. lap. V. p. 62-94, Vl. p. 212-229.

+ Gá y er Di. G, Vorarbeiten zu einer Monographie der europäischen Aconitum Arten.

M. bot. lap. VIII. p. 114-205.

5 Janchen E. Die Cistaceen Oesterreich-Ungarns.

Mith. d. nat. Ver. an der Univ. Wien VIl. p. $1-24$.

" Szabó Dr. Z. De Knautiis Herbarii Dris A. de Degen.

M. bot. lap. IX. p. 1-25.

Krone.

- - Systematische Übersicht der Knautien der Länder der ungar.

Botan. Közlem. 1910 p. 67--99.

- - Monographia generis Knautia.

Mat. és term. közlem. 1911 p. 1-436.

7 Waldstein R. et Kitaibel P. Descriptiones et Icones plant. raz. Hungariae. Viennae 1802-1812.

s Posharsky G. A. Beiträge zur Flora von Croatien und Dalmatien. Dresdener Flora 1896.

${ }^{9} \mathrm{Beck}$ Dr. G. von Mannagetta. Die Vegetationsverhältnisse der illyrischen Länder. Leipzig 1901.

${ }^{10}$ Ross i L. Beiträge zur Kenntniss der Pteridophyten Süd-Kroatiens.

M. bot. lap. X. p. 22-38.

${ }^{11}$ Hacquet B. Phys. polit. Reise aus den dinarischen durch die die julischen, carnischen, rhaetischen und die norischen Alpen in den Jahren 1781 und 1783. Leipzig 1785. 
Uzhkae sa Resnik planine, Popine i brda Gromile te Thymus Serpyllum iz Zrmanje.

Da su ovi krajevi u florističkom pogledu zaista veoma zanimivi osvjedočili smo se tokom naših istraživanja, nu trebalo bi ih jošte pohoditi u razno godišnje doba, da dobijemo podpunu sliku njihovog bogatstva. Nu kako nema iz prije pomenutih razloga izgleda, da će se botanici odvažiti na proučavanje flore ovih krajeva, to smo odlučili, da ovime naš dosadašnji trud oko izučavanja predamo javnosti.

Pretraženo područje obuhvatalo je od južne Plješivice: Bukovi Vrh 1401 kod Bruvna, Orlova Greda i Lisac 1336 kod Glogova i Poštak 1425, zatim Zrmanjska dolina, okolica Gračaca, Śtikade, Ričice i Cerje te Trovrha 1234 a Ljutoč 935, Velika Vrbica 1180, Ćelavac 1207, Zagaljen, Crnopac 1404, Kita Velebita i Tremzina 1175 u jugoistočnom ogranku Velebita, dakle izim donekle Poštaka, Zrmanjske doline, Ploče, Trovrhe i Crnopca sasma nepoznati krajevi.

Zrmanjskom dolinom prošli smo dne 5. travnja 1890. i 7 . srpnja 1901. počam od Vrela do dalmatinske međe. Ovo je dugoljasta plodna kotlina u duljini po prilici od $8 \mathrm{~km}$ a u širini od $2 \mathrm{~km}$ uzdignuta neko $250 \mathrm{~m}$ nad površinom morskom, obrubljena golim krševitim bregovima, a protiče ju rijeka Zrmanja. Podnebje je blago, jer tu uspijeva vinova loza, badem i smokva pa i druge južne biline. Mjeseca travnja brali smo među ostalim kod Vrela: Aethionema saxatile, Arum italicum, Astragalus illyricus, Capsella rubella, Corydalis tenuis, Crocus neapolitanus, Glechoma hirsuta, Hutchinsia petraea, Lamium bifidum, Lithospermum incrassatum, Muscari botryoides, Potentilla micrantha, Rannunculus calthaefolitus. Oko ruševina Rakovnika: Aristolochia pallida, Corydalis densiflora, Geranium purpureum a kod Zvonigrada: Arabis turrita var. glabra, Carex Halleriana, Lamium amplexicaule, Veronica hederifolia, Vicia lathyroides, Viola alba te Cardamine graeca (jedino autentično stanište za Hrvatsku). Od ljetne flore vrijedno je napomenuti: Asperula canescens var. glabra, Centaurea cristata $i$ Fritschii var. spinigera, Silene viridiflora, Sedum ohroleucum, Smyrnium perfoliatum, Melissa officinalis, Vaccaria pyramidata, Stachys serotina var. dalmatica, Scolymus hispanicus iz okolice Vrela; Allium pulchellum, Bupleurum aristatum, Galeopsis canescens, Lagoseris saneta, Rhamnus 
intermedia, Rosa biserrata sa Rakovnika, Campanula pyramidalis, Clematis flammula var. fragrans, Linaria italica, Paliurus spina Christi od Zvonigrada a Acer obtusatum i Potentilla australis od Palanke.

Sjeveroistočno Vrela diže se 1425 m visoki Poštak (a ne Postak $^{1}$. Kada se polazi nanj, prenoći se u cestarskoj kući u Ljeskovoj drazi naprotiv Velike Popine, kao što i mi učinismo za našeg pohoda dne 3. kolovoza 1896. i 8. srpnja 1901. Tu smo jošte pred sumrak u bližnjoj okolini ubrali pored ostalog Centaurea Weldeniana, Euphorbia exigua i falcata, Herniaria hirsuta, Linum tenuifolum, Minuartia fasciculata, Plantago carinata, Thesium divaricatum.

Iz Ljeskove Drage pošli smo zorom preko Osoja brijega i spustili se u ugarska (valjda ugljarska, jer su nekad tamo drveni ugalj zgotavljali). Korita, gdje imade 5 bunara za blago a za piće Vodar bunar, sve sama živa vrela, kod kojih ubrasmo lijepe primjerke od Viola tricolor, onda preko Javorja i Grla prispjesmo na Ljubine Poljane sjeverozapadno Poštaka, na kojim ima izvor vrelo Točak zvano. Ljubine Poljane su gorske livade i optočene kao i cij̣eli sjeverni kraj Poštaka debelom bukovom šumom, dočim je njegova južna strana sasvim gola. Ove prostrane, $1100-1200 \mathrm{~m}$ visoke poljane kriju mnoge rijetke biline poimence; Gentiana crispata, koja tu raste u velikoj množini, zatim Aconitum anthora, Alectorolophus augustifolius, Carduus candicans i alpestris, Cirsium pannonicum, Danthonia calycina, Dianthus velebiticus, Gentiana tergestina $i$ utriculosa, Geranium macrorrhizum, Onobrychis Tommasiniana, Heracleum elegans, Pedicularis Hoermanniana, Phleum phleoides, Stipa pulcherrima, Scorzonera rosea i t. d.

Pregledavši Ljubine Poljane vratismo se istim putem natrag do pod Repino brdo jugozapadno Poštaka. Uspinjuć se prema potonjem po kamenitim pašnjacima ubrasmo: Agropyrum intermedium, Allium pulchellum i sphaerocephalum, Campanula pinifolia i velebitica, Centaurea variegata var. adscendens, Cerastium grandiflorum, Cotoneaster parvifrons, Fumana procumbens, Galium lucidum i purpureum, Genista silvestris, Globularia cordi-

${ }^{1}$ Dobio je ime po straži = Posten, pošta, koja se je na njemu u stara vremena podržavala. 
folia, Helianthemum alpestre forma balcanum i hirtum, Inula ensifolia i hirta, Knautia purpurea, Leontodon hispidus, Minuartia liniflora, Ranunculus sentatus, Rhamnus saxatilis, Rosa gentilis i spinosissima, Ruta clivarita, Scrophularia laciniata, Stachys subcrenata var. eriostachya i velebitica, Avenastrum pubescens, Trinia longipes, Teucrium montanum i mnoge druge.

Prije nego li ćemo se početi verati na vršak Poštaka iznenadiše nas kod nekih pećina: Achillea clavenae, Edraianthus graminifolius, Heliosperma pusillum, Hieracium adriaticum, humile var. sarajevöense i villosum forma amplecissimum, Juniperus nana, Paronychia Kapela, Potentilla Clusiana, Satureia croatica i subspicata, Scabiosa graminifolia i silenifolia, Silene multicaulis i t. d. Od ovud vodi strm kameniti uspon preko pašnjaka na glavicu Poštaka sa raznovrstnim biljkama kao: Allium globosum i montanum, Androsace villosa veoma mnogobrojna, Anthyllis Jacquini, Arctostaphylos uva ursi, Artemisia Lobelii, Bupleur.um Sibthorpianum, Coronilla vaginalis, Dianthus bebius i inodorus, Euphrasia salisburgensis var. adscendens, Sempervivum Schlehani, Thlaspi praecox i druge.

Na povratku s južne strane do ispod Repinog brda ubrasmo još pojedince Ruta patavina, koja raste kod Cerovca u obilnom broju, zatim Seleranthus uncinnatus i Nepeta pannonica. Silazeć mimo Budimirovih kuća dospijemo u Sučević na vispoljanu odkud ponijesmo Thesium divaricatum i Euphrasia illyrica.

Na 26. srpnja 1896. pohodili smo Trovrhu 1234 kod sela Komića sjeverozapadno Gračaca. Put vodi od sela dobrom golom strminom do sedla između Komače i Trovrha a onda krenusmo na desno krčeć si sami put kroz šikarje i grmlje te penjuć se preko hridina i pećina. Uz često odmaranje uslijed napornog uspinjanja stigosmo napokon na goli klisurasti i raspucani vrh Trovrhe. Nakon kratkog odmora vratismo se sjevernom stranom nizbrdice hladovinom, čestoputa spuštajuć se naledjice niz okomite pećine, dok napokon ne stigosmo na podnožje. Trovrha je u opće pokrita gustom bukovom šumom, samo su vrh i nekoji dijelovi na sjevernoj strani ogoljeni. Ma da smo se na ovom uspinjanju pošteno uznojili i umorili naplatio nam se trud obilnim zastupnicima božice Flore.

U okolici sela Komića pribrali smo: Acer campestre var. austriacum forma Bierbachii, Allium pulchelum, Bupleurmm ari- 
statum var. nanum, Chaerophyllum bulbosum, Euphrasia tatarica, Heracleum ternatum, Mentha mollissima, Teucrium botrys a na Trovrhu između ostalog: Tilia flava, Acer monspessulanum, obtusatum i platanoides, Allium sphaerocephalum, Anthriscus fumariaides, Arabis sagittata, Asparagus tennifolius, Bupleurum junceum, Campanula pyramidalis i Waldsteiniana, Centaurea deusta, Daphne alpina var. petiolaris, Digitalis laevigata, Erigeron polymorphus, Evonymus latifolia, Ferulago galbanifera, Hieracium integrifolium, flexuosum i lanifolium, Laburnum alpinum, Laserpitium latifolium var. asperum i glabrum, Physospermum verticillatum, Prunus mahaleb, Rosa ferruginea i praerupticola, Satureia croatica, Sentellaria altissima, Seseli elatum, Spiraea cana, Stachys velebitica var. hirticalyx, Trifolium pratense var. pilosum i ochroleucum.

$\mathrm{Na}$ Klancu povrh Ploče na zapadnoj strani Trovrhe našli smo obje prije pomenute Campanule zatim Digitalis ferruginea, Helleborus multifidus, Inula oculus Christi, Rubus tomentosus, Lactuca Chaiscii, Mentha mollissima, Rhamnus fallax, Sedum album; Verbascum fiuminense takoder sa bijelim cvijetom Thymus subcitratus i druge.

Još smo se god. 1896. uspeli na Crnopac $1404^{1}$ u jugoistočnom Velebitu. Zorom 31. srpnja krenusmo iz Gračaca gračackim poljem na Crljene Potoke mimo Vrataca uzev uz put Mirtuartia fasciculata i Mentha cuspidata, krai Dolova polagano uzdižuć se do Gaćešina stana, gdje imade bunar vode hladne i gdje opazismo ponajprije Juniperus sabina. Onda zakrenusmo na lijevo u guduru idući neprestance uzbrdo hladovinom mimo pećina i hridina sa raznovrsnom florom kao; Dryopteris pallida, Polystichum lonchitis, Lilium Cattaniae. Salix grandifolia var. velebitica, Paeonia mas var. pubescens, Aconitum vulparia var. velebiticum, Kernera saxatilis, Ribes alpinum var. pallidigemnum, Cotoneaster tomentosa i integerrima var. parvifrons, Rosa glaucescens, pendulina i gentilis var. adenoneura, Ruta divaricata, Rhamnus saxatilis i fallax, Daphne alpuna var. petiolaris, Physospermum verticillatum, Athamanta Haynaldi, Cnidium silai-

' Ja sam bio u Gračacu najmanje 30 puta te sam svakom zgodom raspitivao ljude raznih staleža $i$ dobe starosti, pak sam svaki puta dobio odgovor "Crnopac". Neznam kako je pokojni Devčić došao do imena "Srnopas“, kojega tamošnji narod nepoznaje. 
folium, Myosotis suaveolens, Onosma Javorkae, Teucrium Arduini, Satureia variegata, Thymus acicularis var. subalpinus, Scabiosa graminifolia, Campanula Waldsteiniana i pinifolia, Senecio cacaliaster, Carduus alpestris, Hieracium bifidum i t. d.

Nakon skoro jedne ure hoda izlazi se iz ove gudure na sasma goli predjel pa prešav neki brežčić spušta se na južnu stranu Crnopca bezputice idući preko hridina i pećina mimo svakakvih škrapa i rupa, dok se napokon nestigne pod sam vrh. Pred nama ukaza se sada velika strmina bez ikakve staze, ali buduć nebijaše drugog izlaza svladasmo velikim naporom i ovaj tegotni komad uspevši se napokon na najvišu točku blizu kamene piramide.

Do podnožja ubrali smo: Potentilla pedata, Libanotis niteus, Primula Columnae, Satureia thymifolia, Galium lucidum, Edraianthus tenuifolius, Gentiana cruciata, Hieracium flexuosum a tečajem uspinjanja na glavicu nadjosmo novu vrst: Leontodon, kojega prozvaše Rossianus ${ }^{1}$.

Tjemenica njegova je gola i pokrita ogromnimi stijenami, klisurami i pećinami, koje smo marljivo pretražili osobito radi runolista (Leontopodiun alpinum), nu sav trud bijaše nam uzaludan, akoprem ga je kašnje iza nas ipak Janchen našao. Dr Mane Trbojević bivši kotarski liječnik " Gračacu, kazivao nam je, da je on vidio runolist sa Crnopca $\mathrm{i}$ da je nešto manjeg struka nego li onaj sa Schneeberga kod Beča. Ali kad smo ga zamoliti, da nam ga pošalje na ogled, nije to ipak učinio. Vodlić Božo Stojsavljević inače strastven lovac poznavajuć svaki kut. Crnopca, veli, da nije vidio na njem runolist, koga mu takodjer predočismo. Mora dakle da raste samo na stanovitom mjestu i da je veoma rijedak.

Na vrhu smo našli: Festuca pungens, Allium fuscum, Silene multicaulis, Cerastium grandiflorum, Arenaria gracilis, Aquilegia Kitaibelii, Sempervivum Schlehani, Saxifraga Malyi, Spiraea cana, Bupleurum Sibthorpianum, Pimpinella! alpina, Gentiana symphandra, Satureia croatica, Edraia nthus graminifolius, Achillea clavenae, Senecio doronicum, Hieracium humile var. sarajevöense i t. d.

Crnopac je na sjevernoj (hrvatskoj) strani pokrit gustom bukovom šumom, dočim je južna (dalmatinska) posvema gola

1 Degen Dr. A. de et Lengyel. Leontodon Rossianus.

M. bot. lap. IX. p.91-93. 
Da upotpunimo naša dosadašnja istraživanja poduzesmo god. 1911. poseban izlet u ove krajeve sa sjedištem u Gračacu, na 14 dana. Dne 20. srpnja otputovasmo kolima iz Gospića preko Medka, Raduča, Cerja, Ričice i Štikade u Gračac, zabijježiv pri tom množinu opaženih bilina. Odmah sutradan obadjosmo bližnju okolicu Gračaca. Uz desnu obalu Otuče više katoličke crkve, prama sjeveru prostiru se kameniti i pećinasti obronci Resnik planine, koje je kiša i bura malne sasvim isprala ali su raznovrstnim stabaljem i grmljem pokriveni, tako da čine mali gaj u kom nalaziš: Acer campestre, Ostrya carpinifolia, Carpinus duniensis, Omercus vrsti Fraxinus ornus, Ulmus scabra, Prunus mahaleb, Coryius avellana, Ligustrum vulgare, Ribes aldinum var. pallidigemmum, Lonicera xylosteum, Pirus piraster, Evonymus verrucosa i druge. Niže katoličke crkve ubrasmo: Marrubium candidissimum, Scolymus hispanicus, Helleborus multifidus i Trifoliam dalmaticum, koja tu u velikoj množini pridolazi - a u samom gaju još: Corydalis ochroleuca, Poterium polygamum, Aslragalus glycyphyllos, Áremonia agrimonioides, Dianthus armeria, Stachys recta, Melampyrum vulgatum, na jednoj pako ivadi uz potok Bašinicu med grmljem Apopanax chironium, Dianthus velebiticus, Achillea virescens, Asparagus tennifolius, Veronica Jacquini. Od ovuda krenusmo lijevo na obližnji brijeg Batovar $707 \mathrm{~m}$, koj je inače gol, dočim je prama vrhu ponešto šikarjem obrašten uza to jako pećinast. Na njegovom podnožịu raste Trifolium dalmaticum, nu redje, zatim Bupleurum aristatum var. nanum, Eryngium amethystinum, Cirsum acaule, Inula oculus Christi, Centaurium pulchellum, Knautia dissecta i druge. Medju pećinama opazili smo Scrophularia laciniata, Satureia thymifolia, Saxifraga lasiophylla, Prunus mahaleb, Peltaria alliacea, Geranium macrorrhizum, Ribes pallidigemmum, Campanula pyramidalis, Solidago alpesiris, Cotinus coggygria, Digitatis laevigata, Carduus candicaus a na samom vrhu Spiraea cana u rijetkim primjercima.

Sliedećeg dana t. j. 22. srpnja uputismo se na davno željkovanu Tremzinu 1175, u davnini Vučjak zvana, u jugoistočnom dijelu Velebita. Odvezosmo se kolima na istok do 7 . kilometra blizu zaselka Cerovac. Tamošnji kameniti pašnjaci dali su nam izmedu ostalog Satureia thymifolia, Verbascum Chaixii, Globularia Willkommii, Teucrium montanum, Galium purpureum, Plantago carinata, Dorycnium germanicum, Inula ensifolia, Linum tennifo- 
lium, Veronica spicata, Knautia dissecta, Dryopteris Robertiana, Brunella grandiflora, Sedum ochroleucum, Bupleurum aristatum, Trifolium dalmaticum, Ruta divaricata i patavina.

Sabiruć tako idjasmo prama jugu sve niže i niže dok nedospjesmo u dol na podnožje Tremzine otkuda je valjalo poći uzbrdice i to gustom bukovom šumom ne baš preteškim uspinjanjem, dok neizadjosmo na goli hrbat, sastojeći se ponajvećma od kamenitih gorskih livada, koje se više gore prama vrhu izmjenjuju pašnjacima. Vrh sam jest hridovit nu obrašten šumom, dalmatinska strana jest pusta i gola. Na Tremzinu prispjeli smo upravo pravodobno, jer su baš taj dan počeli kositi travu i tako spasismo mnogu bilinu za našu sbirku. Od množine ostalih napominjemo: Hypericum alpigenum, Helianthemum italicum, Centaurea variegata, Inula ensifolia, Teucrium montanum, Rosa gentilis, Scrophularia laciniata, Lilium Cattaniae i Jankae, Sesleria tennifolia, Rumex arifolius, Genista sagittalis i dalmatica, Trifolium montanum i alpestre, Thesium bavarum, Stachys subcrenata var. angustlfolia, Knautia illyrica, Digitalis ambigua, Cynoglossum velebiticum, Bupleurum Sibthorpianum, Cantaurea Haynaldi, Hypochaeris illyrica, Leontodon crispus, Plantago lanceolata var. capitellata, Hieracium cymosum ssp. viridans i Wiesbaurianalm, Orobanche alba, Globularia bellidifolia, Euphrasia liburnica, Campanula Scheuchreri, pinifolia i elliptica, Edraianthus tennifolius.

Zapadno Tremzine leži Crnopac. Tamošnji narod razglaba ga u Kitu Velebita, Bat, što bi odgovaralo visini 1386 i Sljeme t. j. vrh 1404. Dne 23. srpnja pošao sam na Kitu Velebita i to na pećine južno nje, gdje stoji u specijalnoj karti ubilješeno „Lisina”. Iz Gračaca pošli smo preko Glavice na Gaćešinu Jarugu a onda smo mahom zašli u bukovu šumu te krivudajuć amo tamo, da nam put bude što udobniji penjasmo se n'prestano uzbrdice. Tek pred samom Kitom nastadoše znatnije poteškoće, jer samo uzajamnom pripomoći mogosmo se na klisure popeti. Cijelim putem pratila nas je šuma, koja je mjestimice imala male čistine pune raznolikog bilja: Sanicula europaea, Sorbus aria var. Lanifera, Lilium Cattaniae, Prenanthes purpurea, Melittis albida, Elymus europaeus, Asarum europaeum, Senecio Fuchsii, Hypericum hirsutum, Hieracium silvaticum ssp. bifidiforme, Valeriana tripteris var. velebitica, Campanula Waldsteiniana, Ribes grossularia $i$ pallidigemmum, Rubus idaeus, Dryopteris rigida var. 
meridionalis, Malva moschata i var. dissecta, Prunus mahaleb, Solidago alpestris, Thalictrum aquilegifolium.

Oko pećina na podnožju vrška spravismo Heracleum ternatum, Saxifraga Malyi, Cirsium erisathales, Arabis alpina, Aspidium lonchitis, Geranium macrorrhizum, Calamintha alpina, Gentiana symphandra, Heliosperma pusillum, Phyteuma orbiculare ssp. flexuosum, Achillea clavenae, Daphne mezereum i alpinum, Actaea spicata, Asplenium viride, Scabiosa graminifolia, Chamaenerion augustifolium, na samom pak vrhu: Edraianthus graminifolius, Anthyllis Jacquini, Galium lucidum, Ruta divaricata, Cotinus cogsygria, Teucrium montanum, Campanula elliptica, Globularia bellidifolia, Lithospermum officinale, Rosa pendulina spinosissima i var. megalacantha, Vincetoxicum officinale, Amelanchier ovalis, Sesleria tennifolia, Corydalis ochroleuca, Sempervivum Schlehani, Athamanta Haynaldi, Solanum dulcamara i mnoge druge.

Od Kite spustismo se istim pravcem do ispod pećina te zakrenuvši zapadno dodjosmo u Vuč̣u stranu gdje ima živo vrelo "Lokvica" od $16^{0} \mathrm{C}$ dočim je na zraku bilo 22 stupnja. Prama jugu ide se na Bat ali na njegov vršak ne može se dospjeti poradi okomitih golih stijena. Na njem mora da je zanimiva flora sudeć po sabranom bilju oko njegovog podnožja kao: Aquilegia Kitaibelii, Polystichum lonchitis, Sedum roseum, Peltaria alliacea, Onosma Javorkae, Teucrium Arduini, Satureia thymifolia, Senecio Fuchsii, Hieracium flexuosum, Phyteuma orbiculare ssp. flexuosum, Leontodon Rossianus. Povrativši se zatim opet u Vučju stranu te nakon odmora nastavismo put preko Rašlja, što bi odgovaralo visini 775 spec. karte uzev uz put još Melica nutans, Veratrum album, Rubus hirtus, Aquilegia Sternbergii, Trijolium ochrolencum, Linum catharticum, Satureia calamintha var. intermedia, Phyteuma spicatum, Cirsium acaule te preko Crljenih potoka i Vrataca stignemo pred večer u Gračac.

Sljedećih dana nastavismo istraživanjem nekojih zapadno Crnopca ležećih brijegova. Dne 1. kolovoza idući iz Gračaca do Vrataca a onda jugozapadno stranom golom stranom obraštenom strminom dospijemo u malu uvalu sá lijepom jur pokośenom livadom kod Vode Vrbice. Kod Vrataca ubrasmo Stachy's obliqua i Silene Reichenbachiana a povrh njih Herniaria incana, Satureia variegata i thymifolia, Geranium macrorrhizum, 
Juniperus sabina a kod Vode Vrbice Veratrum album, Hypochaeris illyrica, Blysmus compressus, Carex Oederi i nekoje druge, Od Vode Vrbice uspinje se neprestance pustimi pašnjaci do bezimenog vrela a hladnom pitkom vodom od $12^{\circ}$ Celsija, više kojeg započima bukova šuma, kojom idemo preko pol sata dosta strmo, dok neizadjemo na čistinu ugledavši pred sobom blizi goli vrh Velike Vrbice $1180 \mathrm{~m}$. Sa njega gledamo prama jugozapadu dugu golu kamenitu kotlinu Male i Duboke Jasle a a onda opet goli kameniti bedem velebitske kose sa raznimi okomitimi i nepristupnimi vrhovi od $900-1000 \mathrm{~m}$. Ovu kotlinu valjalo bi svakako u ranije godišnje doba pohoditi, jer smo uvjereni, da bi se našlo mnogo zastupnika južne flore. Veliku Vrbicu i bližnji Celovac pokrivaju na hrvatskoj strani bukove šume, dočim je dalmatinska strana gola $i$ jako krševita. Oko vrha Velike Vrbice prostirući se kameniti pašnjaci obiluju bujnom florom kao: Cynoglossum velebiticum, Peucedanum montanum, Iuniperus nana, Primula suaveolens, Veronica spicata, Rosa gentilis, Galium lucidum, Inula ensijolia, Teucrium montanum, Saxifraga Malyi, Campanula Waldsteiniana, Sedum ochroleucum, Valeriana tripteris var. velebitica, Ruta divaricata, Cerastium grandiflorum, Iuniperus sabina, Rhamnus rupestris, Scrophularia laciniata, Ribes alpinum var. pallidigemnium, Contaurea variegata, Prunus mahaleb, Veronica Jacquini, Asphodelus albus, Delphinium fissum var. velebiticum i t. d. i t. d.

Idıći većim dijelom po sljemenu prema jogoistoku dospijemo do Ćelavca 1207 te na ledinama oko njegova vrha sabrasmo lijep struk bilina: Sesleria tennifolia, Anthyllis Jacquini, Athamanta Haynaldi, Sempervivum Schlehani, Satureia thymifolia i croatica, Sedum album i ochroleucum, Iuniperus nana, Rhamnus saxatilis, Ribes grossularia i pallidigemmum, Veronica spicata, Inula ensifolia, Ruta divaricata, Cynoglossum velebiticum, Thlaspi praecox, Scutellaria altissima, Arabis hirsuta, Geum urbanum, Moehringia muscosa, Scrophularia laciniata a na njegovom drugom vrhu povrh napomenutih još: Cymbalaria muralis, $S a-$ xifraga lasiophylla, Lonicera alpigena, Campanula Waldsteiniana, Senecio rupestris, Valeriana tripteris var. velebitica, Arabis alpina, Spiraea cana mnogo, Silene multicaulis, Saxifraga Malyi, Sedum maximum, Teucrium Arduini, Paeonia mas var. pubescens, Artemisia Lobelii, Asphodelus albus, Stachys germanica i mnoge druge. 
Kada smo ovako Ćelavac svestrano pretražili spustismo se južnom dosta strmom i kamenitom stranom u dolinu, a onda uzbrdo na takoder goli od Čelavca južno ležeći Zagaljen, koji. u specijalnoj karti nije imenom označen. Njegova je flora veoma zanimiva pak navađamo samo: Iuniperus sabina i nana, Teucrinm Arduini, Campanula Waldsteiniana, Satureia variegata, thymifolia i croatica, Daplme alpina var. petiolaris, Ruta divaricata, Silene multicaulis, Cynoglossum velebiticum, Senecio rupestris.

Ovime svršismo današnju exkursiju i vratismo se istočnom stranom kući uz dodatak, da smo ispocl Ćelavca našli čitavu šumu aptovine (Sambucus ebulus) " tako visokim primjercima, kakovilh u čitavoj Hrvatskoj dosele opazili nismo a nad Dolovima Geranium macrorrhizum u neobičnoj množini na okupu.

I'onačno smo se još dne 3. kolovoza otputili na Ljutoč 935 m. ležeći sjevero-zapadno Velike Vrbice. Kolima podosmo iz Gračaca kroz Štikadu, gdje opazismo Stachys obliqua u veśem broju, do Bunara t. j. vrela optočena šupljim panjem na otvorenom suncu izvrženom prostoru sa $15^{\circ} \mathrm{C}$, nedaleko Gudure sela a onda pješke kroz selo jugozapadnim puteljkom neprestano uzbrdice. Više vrela Korito zvana sa $9^{0} \mathrm{C}$ na laporastom mjestu ubrasmo Hieracium silvaticum spp. oblongum a malo više ispod jednog kuka Digitalis laevigata, Arabis turrita, Phyteuma orbiculare ssp. flexulosum, Sedum maximum, Lonicera alpigena, Campanula Waldsteiniana Satureia croatica u visini po prilici od $800 \mathrm{~m}$. Sašav na put opazismo žene kako nose breme granja od žestikovine (R/amnus fallax), koje nam na upit odvratiše, da lišćem hrane ovce preko zime. Pošto smo obišli zapadnu stranu krenusmo prama sjeveru kamenitom bukovom šumom sa mnogimi čistinami obilujućimi svakovrsnimi bilinami kao: Digitalis ferruginea, Peucedanum montanum, Aquilegia Sternbergii, Bupleurum aristatum var. nanum, Campanula pyramidalis, Senecio rupestris i t. d. Sam uzlaz na vrh jest dosta mučan, jer valja preko velikih strmina i kamenitih ploča prelaziti, nu mapokon je $\mathrm{i}$ to obavljeno, pa sa takove jedne klisure promatraš okolinu, koja je izim malog dijela vrška pokrita raznim drvećem. Tu nam pada u oči jedno 10 komada stabala punih žuto crvenog grozdastog ploda a to je jasika (Sorbus aucuparia) zatim lijepe bukve, Ostrya carpinifolia, Rhamnus fallax, Acer pseudoplatanus i obtusatum, pa Spiraea cana (mnogo- 
brojno). Od ostalog cvijeća neka bude napomenuto iz bližnje okolice vrha: Lonicera alpigena, Campanula Waldsteiniana i pyramidalis, Peltaria alliacea, Scrophularia laciniata, Rubus idaeus, Cystopteris fragilis, Polystichum lobatum, Chamaencrion angustifolium, Scrophularia laciniata, Valeriana Iripteris var. velebitica, Satureia thymifolia i variegata, Malva dissecta, Achillea virescens, Ornithogalum pyramidale.

Sa vrha spustismo se južnom stranom prama istoku a zatim zaokrenuv dospijemo na sjeverni kraj Ljutoča otkuda uputismo se u Guduru ponesav sobom između ostaloga još: Rosa rubrifolia i badensis, Pimpinella major i saxifraga, Artemisia Lobelii, Vulpia myurus, Convolvulus arvensis var. Cherleri, Moenchia mantica, Mentha candicans var. stenanthelmla.

Pošto smo ovom zgodom odlučili bili da i nekoje brijegove južne Plješivice pohodimo upriličismo u tu svrhu jedan dvodnevni izlet. Dne 27. kolovoza 1911. pođosmo iz Gračaca put istoka do 3. kilometra. Kod Kesića kuća zakrenusino sjeverno stazom mimo brda Kamare 804 Drobac kuća i dođosmo nad Glogovo, gdje se kod vrela Točak od $9^{\circ} \mathrm{C}$ zaustavismo. Sama kamenita pusta okolica mjestimice sa malo težatne zemlje crljenice! Putem opazismo: Marrubium vulgare i candissimum, Anthericum ramosum, Galium purpureum, Globularia cordifolia, Scorzonera villosa, Inula hirta i ensifolia, Knautia diessecta, Herniaria glabra i t. d. Nedaleko više spomenutog vrela jest spomenik cara Josipa II., naime onizka piramida od klesanog kamena providena na sve četiri strane napisi u hrvatskom, njemačkom i latinskom jeziku of kojih napominjem hrvatski ukljesan na sjevernoj i južnoj strani: „Josip II. pod ovom bukvom u sjeni opočinu dne 7 . svibnja 1775 . - Spomenik ovaj postaviše časnici Ličke pukovnije godine 1858." - Ovu bukvu posjekoše prije 30 godina zlobne ruke.

Sada idemo gorskimi kamenitimi pašnjaci uzdignutimi po prilici $800--900 \mathrm{~m}$ nad površinom morskom neprestance polagano uzdižući se, na kojima mora da raste u ranije doba bogata flora sudeći po nekojim ubranim bilinama kao: Iurinea mollis var. liburnica, Silene otites i pseudotites, Thalictrum simplex, Hypochaeris illyrica, Stachys serotina i t. d. Zaokrenuvši prama sjeveru dođemo na južno podnožje Lisca 1336 okriepivši se sa hladnom vodom iz Borovac vrela od $14^{\circ} \mathrm{C}$, dočim je 
zrak imao $32^{\circ} \mathrm{C}$. Uspon poduzesmo sa zapadne strane kod livade, koje počeše taj dan upravo kositi. Na tom po prilici $1200 \mathrm{~m}$ visokom prostoru ubrasmo medu ostalim: Plantago carinata, Campanula pinifolia, Veronica spicata, Sedum ochroleucum, Globularia cordifolia, Galium purpureum, Saturcia thymifolia, Eryngium amethystinum, Koeleria montana, Asphodelus albus, Knautia dissecta, Gentiana symphyandra, Rosa gentilis, Primula suaveolens, Medicago prostrata, Centaurea variegata i jedan primjerak Cirsium Freyerianum.

Dalnje napredovanje bijaše nam oteščano znatnom strminom uz veliku sunčanu žegu a bez ikakva puteljka, nu napokon popesmo se ipak na vrhunac Lisca, koj je posvema gol, osim nešto grmlja na podnožju zapadne strane. Između Lisca i naprotiv sjeverno ležećeg Bukovog vrha 1401 proteže se do dva kilometra dugo a jedan kilometar široko Pusto Polje ${ }^{1}$ sa lijepimi livadami posuto mnogobrojnimi vreli, koja iz pećina izviraju kao: Zvijezda, Crno vrelo i Vrelo kod strane Maričića sa 8 a Milovanović vrelo i Mala Zvijezda sa $13^{\circ} \mathrm{C}$ te koja svojom vodom također natapaju pomenuto polje. Pri podnevnoj žegi pretražismo mu vrh na sve strane našav nešto ljetne flore, jer smo prekasno došli i to: Athamanta Haynaldi, Euphrasia illyrica, Saxifraga Malyi, Dryopteris rigida, Gentiana utriculosa, Anthyllis Jacquini, Galium lucidum, Stachys subcrenata, Satureia thymifolia, Aspcrula longiflora u niskim patuljastim primjercima.

Nakon odmora spustismo se istočnom položitijom stranom prama pustom polju, pri čem smo našli Gentiana crispata, poznatu nam dosele iz Hrvatske sa Poštaka, Minuartia verna, Dianthus velebiticus i neke druge. U samom Pustom polju nedaleko vrela Zvijezde netom pomenute pa Cirsium eriophorum, Thalictrum simplex, Ornithogalum tenuifolium, na mokrim pako mjestima Blysmus compressus, Iuncus alpinus var. fuscoater, compressus i bufonius, Caltha palustris.

Okriepivši se kod vrela Zvijezde hladnom vodom stanemo se polagano uspinjati na bukovom šumom obrasli Veliki Bukovnik. Nu kako smo sabiranjem i bilježenjem mnogo vremena potratili zateče nas mrak te bijasmo prisiljeni na istočnoj strani

1 Nazvaše ga radi toga, jer na njegovom prostranom prostoru nema nikakve kuće a niti stana. 
V. Bukovnika po prilici u visini od $1250 \mathrm{~m}$ da prenoćimo pod vedrim nebom. Spominjemo nekoje od opaženih bilina kako smo jih redomice zabilịežili: Asphodelus albus, Inula oculus Christi, Globularia Willkommii, Dorycnium germanicum, Gentiana crispata, Orobanche alba, Helianthemum obscurum, Melica ciliata, Galium purpureum i lucidum, Rhamnus fallax, Trifolium alpestre, Euphorbia amygdaloides, Brunella alba, Scrophularia laciniata, Cynoglossum montanum, Geranium robertianum, Thlaspi praecox, Carduls alpestris, Dianthus velebiticus, Allium ursinum, Epilobium montanum, Ranunculus lanuginosus, Veronica pseudochamaedrys, Stachys silvatica, Chenopodium bonus Henricus, Thymus angustifolius, Paris quadrifolia, Aremonia agrimonioides i t. d.

Zorom 28. srpnja prispjesmo nakon napornog uspinjanja preko omašnih pećima na sám goli Bukovi Vrh 1401 pri čem smo u našu mapu pohranili Lonicera alpigena, Sedum album, Dentaria polyphylla, Polystichum lobatum, Convallaria polygorıatum. Stachys alt ina, Geranium macrorrhizum, Peltaria alliacea, Verbascum floccosum, Arabis alpina, Scutellaria altissima, Saxifraga lasiophylla, Chamaenerion angustifolium, dapače nađosmo Urtica divica i Coronilla varia na sámom vrhu. Idući sjeverozapadnim pravcem dospijemo do visine 1330 a spustiv se zatim nizbrdice prama Pustom polju opazismo na jednoj krčevini neizmjernu množinu šumskih jagoda (Fragaria moschata) u cvijetu i plodu, ali veći dio njih tek u dozrijevanju. Do silaza na Pusto polje ponesli smo još: Rumex arifolius, Aegopodium podagraria, Melandryum rubrum, Maianthemum bifolium (u listu) Lilium martagon, Primula suaveolens, Petasites albus, Chrysanthemum macrophyllum, Cirsium pannonicum, Gymnadenia conopea.

Idući napred Pustim Poljem niz potočić Soviljevac dospijemo u klanac, kojega južno ležeću stran sačinjavaju okomite kamenite stijene Orlove Grede. Na nju se ne može s' ovkraj popeti pa smo stoga samo na podnožju njezinom mogli koješta sabrati kao: Silene multicaulis, Ruta divaricata, Rhamnus fallax, Malcolmia serbica, Athamanta Haynaldi, Campanula Waldsteiniana, Arabis alpina, Polystichum rigidum, Sedum ochroleucum i glaucum, Bupleurum Sibthorpianum, Saxifraga Malyi, Ribes grossularia, Geranium macrorrhizum, Moehringia muscosa, Cystopteris fragilis. Medutim o podne nastali tutanj gromova i silna naoblaka na nebu prisili nas na nastavak puta. Nesašav pravo ni 
na stazu počme kiša pljuštiti, ubrzamo stoga naše napredovanje te uspinjuć se postepeno dospiịemo do pod brijeg Sedlo 1270, kojeg bi bili rado radi njegove kamenite konfiguracije pregledali, nu sbog lošeg i nesigurnog vremena moradosmo se spustiti dole prama Tajagić kućam, gdje nam dopane ruku još Stachys obliqua. Od sedla je lijep izgled na 5 kilometara dugo a $3 \mathrm{~km}$. široko te po prilici $750 \mathrm{~m}$. visoko kamenito i pusto Hubačevo polje, kojeg smo od Tajagića prama Omsici u najljepšem vremenu prešli. Ono malo trave što na njem poraste, popasle su već ovce pa si mogao tek nekoje oporitije biline opaziti kao: Cirsium eriophorum, Teucrium montanum, Marrubium candidissimum, Erysimum crysimoides, Helleborus multifidus, Scrophularia canina, Carlina aggregata i vulgaris, Verbascum nigrum, Rumex pulcher, Centaurea scabiosa, Cirsium acaule i argenteum, Inula oculus Christi.

U Omsici držasmo odmor a onda nastavismo put prama Tomıngaju. Iz tamošnje okolice napominjen o povrh gore istaknutilı još Marrubium vulgare, Galium purpureum, Verbascum floccosum i Chaixii, Acer campestre, Potentilla reptans, Asperula longiflora, Dipsacus silvestris, Clematis vitalba, Calamintha officinalis var. intermedia, Anthemis arvensis a od Tomingaja, kojeg smo povrh toga 25. srpnja posebno pohodili: Scrophularia canina, Galium mollugo i verum, Agropyrum repens, Medicago fulcata, Inula britanica, Ononis spinosa floribus albis, Stachys recta, Orlaya grandiflora, Ferulago galbanifera, Smyrnium perfoliatum, Reseda lutea, Bupleurum aristatum, Opopanax chironium, Mentha spicata, Onopordum acantium, Lathyrus megalanthus, Stachys germanica, Buphthalmum salicifolium, Teucrium montanum i t. d. Iz Tomingaja vratismo se na kolima u Gračac.

Konačno zahvaljujemo se g. dru. A. Degenu u Budapešt, i K. H. Zahnu u Karlsruhe, koji su i to prvi mnoge vrsti našega lierbara a drugi naše Hieracije blagohotıo označili, dočim je pokojni dr. Borbás nekoje ruže determinovao. Našim vrijednim pratiocem na izletima gg. veleč. župniku Ljudevitu Barbiću, sudbenom akcesisti Nikoli Radeki i njegovom sinu petoškolcu Milanu, kao i svim inim, koji su naš rothvat ma na koj način podupirali, neka bude ovime izrečena naša najsrdačnija hvala.

U Zagrebu dne 10. lipnja 1913. 


\section{Sistematski popis ${ }^{1}$.}

\section{Pteridophyta.}

\section{Polypodiaceae.}

Cystopteris fragilis (L.) Bern. var. anthriscifolia Koch. Trovrha, Ljutoć, V. Vrbica, Vratce, Crnopac, Kita Velebita, Tremzina.

Dryopteris Filix mas (L.) Schott. forma typica Luerssen. Trovrha, Ljutoč, V. Vrbica, Ćelavac, Kita Velebita, Tremzina. var. incisa Moore na Trovrhi. - Dr. rigida (Hoffm.) Desv. var. bipinnatisecta forma germanica Milde. Ljutoč, Zagaljen, Crnopac, Kita Velebita. - forma meridionalis Milde. V. Vrbica, Ćelavac, Crnopac, Kita Velebita. - Dr. pallida (Bory) Janchen. Crnopac.

Polystichum lonchitis (L.) Roth. Crnopac (Zelebor, Rossi), Kita Velebita i Bat na Crnopcu. - P. lobatum (Hunds.) Presl. Trovrha, Ljutoč, Ćelavac, Kita Velebita.

Athyrium filix femina Roth. var. fissidens Döll. Rašlje na Crnopcu.

Phyllitis Scolopendrium (L.) Newmann. Trovrha, Kita Velebita.

Asplenium viride Huds forma typica Luerss. Trovrha, Kita Velebita. - A trichomanes L, forma typica Luerss. Ričice, Ploča, Trovrha, Ljutoč, V. Vrbica, Ćelavac, Zagaljen, Vratce, Kita Velebita, Tremzina. - var. auriculata Milde. Trovrha. - var. lobatocrenata DC. Crnopac. - A. muzaria L. V. Vrbica, Ljutoč, Kita Vel., Tremzina. - var. Brunfelsii Haufler. Rakovnik, Zagaljen, Tremzina. - var. pseudo-germanicum, Heufler, Ploča.

Ceterach officinarum Lam. et DC. var. stenoloba Gsh. Rakovnik, Vratce, Ljutoč. - var. platyloba Gsh. Zagaljen. - var. spiralis Deg. et Kümm. Trovrha, Vrelo u Zrmanji, Ćelavac, Crnopac.

Pteridium aquilinum (L.) Kuhn. Ričice. - var. lanuginosa Hook. Ljutoč.

Polypodium vulgare L. a) commune Milde. Ljutoč, Ćelavac, Tremzina. - var. rotundatum Milde. Trovrha. - var. attenuatum Milde. Ljutoč. - forma crenatum Baenitz. Crnopac. - var. auritum Willd. forma auritto-attenuatum Baenitz. Trovrha.

1 U ovaj popis nisu poprimljena ona staništa koja su već objelodanjena u mojoj zadnjoj raspravi „Die Plješivica und ihr Verbindungszug mit dem Velebit in bot. Hinsicht (M. bot. lap. 1913. p. 37-106)“.

Ljudevit Rossi: Floristička istraživanja po jugoistočnoj Hrvatskoj. 
Equisetaceae.

Equisetum arvense L. Ričice.

\section{Anthophyta.}

\section{A. Gymnospermae.}

Coniferae.

Abies alba Miller. Pojedince na Kiti Vel.

Juniperus communis L. Kita Vel. - J. nana Willd. V. Verbica, Celavac, Zagaljen, Crnopac, Kita Vel. - J. sabina L. Crnopac (Zelebor, Janchen, Rossi) V. Vrbica, Ćlavac, Zagaljen. Taxus baccata L. Kita Vel. pojedince.

\section{B. Angiospermae.}

Monocotyledoneae.

Typhaceae.

Typha angustifolia L. Ričice.

Alismataceae.

Alisma plantago L. Štikada, Ričice.

Gramnieae.

Echinochloa crus galli (L.) R. et Sch. Štikada. - E. viridis (L.) R. et Sch. Ljutoč, V. Vrbica, Vratce.

Anthoxantum odoratum L. V. Vrbica, Tremzina.

Stipa pulcherrima C. Koh. Zagaljen.

Phleum pratense L. var. nodosum L. Vratce, V. Vrbica, Kita Vel.

Alopecurus pratensis L. Vratce, V. Vrbica, Gudura.

Agrostis alba L. Ljutoč, Vrbica. - A. vulgaris With. Štikada, Ljutoč, V. Vrbica, Vratce, Tremzina.

Calamagrostis epigeios (L.) Roth. Crnopac.

Avenastrum pubescens (Huds.) Jess. V. Vrbica.

Cynodon dactylon (L.) Pers. Vratce, Štikada, Ljutoč.

Sesleria tenuifolia Schrad. V. Vrbica, Ćelavac, Zagaljen, Kita Vel., Tremzina. - S. autumnalis (Scop.) Schltz. Ljutoč, V. Vrbica, Kita Vel. 
Koeleria montana (Hansm.) D. T. V. Vrbica, Zagaljen, Vratce.

Melica ciliata L. Trovrha, Gudura, Ljutoč, V. Vrbica, Célavac, Zagaljen, Vratce, Kita. - M. uniflora Retz. Ljutoč. - M. nutans $L$. Ljutoč i Rašlje na Crnopcu.

Dactylis glomerata L. Ljutoč, V. Vrbica. Kita, Tremzina. Cynosurus cristatus L. Stikada.

Poa annua $L$. Štikada. - P. nemoralis $L$. V. Vrbica, Ćelavac, Crnopac, Kita, Sremzina. - var. coarctata Gand. Trovrha. - P. compressa L. Ljutoč, Kita, Tremzina.

Festuca ovina L. Ljutoč. - F. pungens Kit. Crnopac. F. silvatica (Poll.) Vill. Trovrha.

Vulpia myurus (L.) Gmel. Ljutoč.

Bromus arvensis L. Ričice, Gudura. -- Br. mollis L. var. contractus Lge. Ljutoč. - Br. erectus Hunds. V. Vrbica, Celavac, Kita, Trenızina. - Br. sterilis L. Cerje, Vratce.

Brachypodium pinnatum (L.) P. Beanv. var. rupestre R. et Sch. V. Vrbica. - Br. silvaticum R. et Sch. Ljutoč.

Lolium perenne L. Ljutoč, V. Vrbica, Vratce.

Agropyron intermedium (Host) Beanv. Štikada, Cerje, V. Vrbica. - var. virescens (Panč) V. Vrbica.

Elymus europaeus L. Trovrha, Kita.

\section{Cyperaceae.}

Schenoplectus lacustris (L.) Palla. Štikada. - Blysmus compressus (L.) Panz. Voda Vrbica.

Heleacharis palustris (L.) R. Br. Štikada, Ričice, Gudura.

Carex Leersii F. Schltz. Tremzina. - C. Halieriana Asso. Vrelo i Zvonigrad u Zrmanji. - C. Oederi Retz. Voda Vrbica.

\section{Araceae.}

Arum italicum Mill. Vrelo i Zvonigrad u Zrmanji.

\section{Juncaceae.}

Juncus conglomeratus L. Voda Vrbica. - J. articulutus $L$. Gudura, Ričice. - J. compressus Jacq. Voda Vrbica. - J. bufonius L. Štikada, Gudura, Ričice.

Luzula campestris (L) DC. Ljutoč, Tremzina. 


\section{Liliaceae.}

Veratrum nigrum L. V. Vrbica. - V. album L. Ljutoč, V. Vrbiba, Rašlje na Crnopcu, Tremzina.

Asphodelus albus L. V. Vrbica, Celavac.

Anthericum ramosum L. Trovrha, Crnopac, Tremzina.

Allium sphaerocephalum L. Trovrha, V. Vrbica, Ćelavac, Zagaljen. - A. ochroleucum W. K. Trovrha. - A. carinatum L. Trovrha, V. Vrbica, Vratce. - A. pulchellum Don. Trovrha, Rakovnik, Ljutoč, Ćelavac, Crnopac, Tremzina. - A. fuscum W. K. Zagaljen, Crnopac.

Lilium Martagon L. Ljutoč, Kita, Tremzina - var. Cattaniae Vis. Crnopac, Kita, Tremzina. - L. carniolicum Bernh. 3. Jankae A. Kerner. Tremzina.

Scilla bifolia $L$. Na podnožju Crnopca.

Ornithogalum pyramidale $L$. Ljutoč.

Muscari botryoides (L.) Lam. et DC. Vrelo u Zrmanji.

Asparagus tenuifolius Lam. Trovrha, Tremzina.

Ruscus aculeatus L. Vrelo i Zvonigrad u Zrmanji.

Polygonatum officinale All. Kita, Tremzina.

Paris quadrifolia L. Kita.

\section{Amaryllidaceae.}

Galanthus nivalis $L$. Na podnožju Crnopca.

\section{Iridaceae.}

Crocus neapolitanus (Rez) Asch. Vrelo u Zrmanji, V. Vrbica, na podnožju Crnopca.

Orhidaceae.

Gymnadenia conopea (L.) R. Br. Tremzina.

Cephalanthera rubra (L.) Rich. Kita.

Epipactis latifolia (L.) All. Ljutoč, Kita.

Neottia nidus avis (L.) Rich. Čelavac, Kita.

\section{Dicotyledoneae.}

Salicaceae.

Populus tremula L. Ričice, Ljutoč. - P. nigra L. Cerje. Salix alba L. Cerje, Ričice, Štikada. - S. glabra Scop. Crnopac (Zelobor). - S. purpurea L. Cerje, Ričica, Štikada. - 
S. capraea L. Bat na Crnopcu. - S. grandifolia Ser. var. velebitica Borb. Crnopac.

\section{Betulaceae.}

Carpinus orientalis Mill. Štikada, Rašlje, Tremzina, Trovrha. Ploča (Beck); u Zrmanjskoj dolini kod Vrela, Rakovnika i Zvonigrada.

Ostrya carpinifolia Scop. U Zrmanji (Sabljar) kod Vrela i Zvonigrada, Ljutoč.

Corylus avellana L. V. Vrbica, Ćelavac, Tremzina.

Fagaceae.

Fagus silvatica L. Ljutoč, V. Vrbica, Ćelavac, Zagaljen, Crnopac, Kita, Tremzina.

Quercus cerris L. Cerje, Ljutoč. - Qu. lanuginosa (Lam.) Thuill. Palanka u Zrmanji. - Qu. sessiliflora Salisb. Ploča (Beck), Trovrha.

\section{Ulmaceae.}

Ulmus scabra Mill. Cerje, Kita.

Moraceae.

Ficus carica L. Vrelo i Zvonigrad, culta.

Urticaceae.

Urtica dioica L. Štikada, Gudura, Ćelavac, Vratce.

Santalaceae.

Thesium bavarum Schrk. Tremzina.

Aristolochiaceae.

Asarum europaeum L: Crnopac, Kita. Aristolochia pallida Willd. Vrelo u Zrmanji.

Polygonaceae.

Rumex obtusifolius $L$. Vratce, Štikada. - R. acetosella $L$. Štikada, V. Vrbica, Tremzina. - R. arifolius All. Tremzina. $R$. acetosa $L$. Štikada, Tremzina. 
Polygonum persicaria L. Štikada, Ričice. - P. aviculare L. Štikada, Ljutoč, Vratce.

\section{Chenopodiaceae.}

Polycnemum arvense L. Vratce.

Chenopoditum vulvaria L. Trovrha. - Ch. album L. Štikada.

\section{Amarantacea.}

Amarantus retroflexus L. Gudura.

Caryophyllaceae.

Agrostemma githago L. Štikada, Gudura.

Silene vulgaris (Moench.) Garcke. Cerje, Ljutoč, V. Vrbica, Vratce, Kita. - S. saponariaefolia Schott. Ljutoč. - S. multicaulis Guss. Čelavac, Zagaljen, Crnopac, Kita. - S. nutans L. Ljutoč, Tremzina. - S. viridiflora $L$. Vrelo u Zrmanji. - S. Reichenbachiana Vis. Vratce, Celavac. Ovamo ce valjda spadati i S. flavescens $W . K$. sa Trovrhe (Zelebor).

Heliosperma pusillum W. K. Crnopac (Zelebor, Rossi), Kita, Tremzina.

Melandryum album (Mill.) Garcke. Vratce, Štikada. Ričice, Cerje, Ljutoč. - M. rubrum (Schk.) Röhl. V. Vrbica, Ćelavac.

Tunica saxifraga (L.) Scop. Vrelo i Rakovnik u Zrmanji, Štikada, Ljutoč, V. Vrbica, Vratce, Kita, Tremzina.

Vaccaria pyramidata Med. Vrelo u Zrmanji.

Dianthus armeria L. Ljutoč. - D. velebiticus Borb. V. Vrbica, Tremzina.

Saponaria officinalis $L$. Vrelo u Zrmanji, Štikada.

Stelları media (L.) Vrelo u Zrmanji. St. glochidisperma Murb. Ćelavac. - St. holostea L. Zvonigrad. - St. graminea L. Na podini Tremzine.

Cerastium semidecandrum L. Vrelo u Zrmanji. - C. ciliatum W. K. Tremzina. - C. grandiflorum W. K. Crnopac (Zelebor, Rossi), Kita, V. Vrbica.

Moenchia mantica (L.) Bartl., Gudura.

Sagina procumbens $L$. Štikada.

Minuartia liniflora (L. fil.) Schinz et Thell. Vrelo u Zrmanji (Stur exicc.). - M. fasciculata Hiern. Crnopac. 
Arenaria serpyllifolia L. Vrelo u Zrmanji, Ričice, Ljutoč, V. Vrbica, Ćelavic, Zagaljen, Vratce, Kita, Tremzina. - A. gracilis W.K. Crnopac (Zelebor, Rossi).

Moehringia muscosa L. Trovrha, Ljutoč, V. Vrbica, Ćelavac, Kita, Crnopac, Tremzina. - M. trinervia (L.) Clairv. V. Vrbica.

Spergula arvensis L. Selo Gudura kod Stikade.

Herniaria glabra L. Trovrha, V. vrbica, Crnopac, Tremzina. - H. incana Lam. Zrmanja (Šloser i Vukotinović exsicc. 1878.) izmedju Vrataca i Vode Vrbice, rijetka.

Scleranthus annuus L. Štikada. Na Ćelavcu ubrao sam novu vrst koja će biti upisana u Degenovoj flori velebitskoj.

\section{Ranunculaceae.}

Paeonia mas Gars. var. pubescens Vis. Ćelavac. Crnopac, Kita.

Helleborus odorus W. K. var. istriacus Schiffn. na podnožju Crnopca. - H. multifidus Vis. Štikada, Gudura, Ričice, Cerje, Ljutoč, Vratce, Crnopac, Tremzina. - H. atrorubens W. K. Ploča.

Isopyrum thalictroides $L$. Vrelo u Zrmanji.

Actaea spicata L. Kita.

Aquilegia Kitaibelii Schott. Crnopac (Zelebor, Jancken, Rossi), Bat na Crnopcu. - A. Sterbergii Rchb. Ljutoč, Rašlje na Crnopcu.

Delphinium fissum W. K. var. dinaricum Beck. V. Vrbica nadjen samo 1 komad!

Aconitum anthora L. V. Vrbica. - A. vulparia Rchb. var. croaticum Gáyer. Crnopac, Kita (Degen). - forma velebiticum Gáyer. Kita (Degen), Crnopac.

Anemone grandis (Wender.) Kern. Tremzina. Ovu mi je bilinu donio 3, travija 1890 tadanji županijski tajnik g. Varga. Ja sam ju brao prije togo kod Mamutovca povrh Karlobaga, gdje bijaše mnogobrojnija. - A. ranunculoides $L$. Vrelo u Zrmanji, Kita. - A. nemorosa L. Vrelo i Zvonigrad u Zrmanji, V. Vrbica, Ćelavac, Kita.

Clematis recta $L$. Ćelavac. - Cl. vitalba $L$. Štikada, Ričice, Ljutoč. - Cl. flammula L. var. fragrans Ten. Vrelo u Zrmanji.

Ranunculus calthaefolius (Rchb.) Bl. N. et Sch. Vrelo i Zvonigrad u Zrmanji. - R. bulbosus L. Cerje, V. Vrbica. $R$. sardous Crtz. var. mediterraneus Griseb. Gudura. - R. re 
pens L. Štikada, Gudura, Ričice. - R. lanuginosus L. Crnopac, Tremzina. $-R$. acer $L$. Voda Vrbica. $-R$. tenuifolius $D C$. var. velebiticus Degen. Kita.

Thalictrum aquilegifolium L. Ćelavac, Kita. - Th. elatum Jacq. Štikada, Ričice, V. Vrbica, Tremzina.

\section{Papaveraceae.}

Chelidonium maius L. Rakovnik u Zrmanji.

Papaver rhoeas $L$. Vrelo u Zrmanji, Śtikada.

Corydalis solida (L.) Schw. Vrelo u Zrmanji. - C. densiflora Presl. Vrelo i Rakovnik u Zrmanji. „Von der südlichen (ital. griechischen) Pflanze nur durch die mehr grüne Farbe des Laubes verschieden. Dr. Degen in sched." - C. tennis Schott. Vrelo u Zrmanji. - C. ochroleuca Koch. Ploča. (Kitaibel, Fl. cr. kao Fumaria odnosno Corydalis capnoides, Rossi), Crnopac, Kita Velebita.

\section{Cruciferae.}

Aethionema saxatile (L.) R. Br. Trovrha, Vrelo i Zvonigrad u Zrmanji, Ljutoč, V. Vrbica, Celavac.

Thlaspi perfoliatum L. ß.) dentatum Borb. Vrelo u Zrmanji. - T. praccox Wulf. V. Vrbica, Ćelavac, Tremzina.

Kernera saxatilis (L.) Rchb. Crnopac, Tremzina.

Peltaria alliacea L. Trovrha, Ljutoč, V. Vrbica, Ćelavac, Zagaljen, Crnopac, Bat, Kita, Tremzina.

Alliaria officinalis Andrz. Zvonigrad.

Sisymbrium officinale (L.) Scop. Vrelo i Rakovnik u Zrmanji, Gudura.

Sinapis arvensis L. Štikada, Ričice.

Diplotaxis tenuifolia (L.) DC. Vrelo i Zvonigrad u Zrmanji. Raphanus raphanistrum L. Ričice.

Barbarae vulgaris L. Gudura.

Roripa silvestris (L.) Bess. Štikada, Gudura, Ričice.

Armoracia rusticana (Lamı.) G. M. Sch. Ričice.

Cardamine graeca $L$. Pod Zvonigradom kraj ceste na obraslom kamenitom tlu dosta mnogobrojna. Jedino autentično nalazište za Hrvatsku. C. hirsuta L. Vrelo i Zvonigrad u Zrmanji. Ćelavac. - C. enneaphyllos (L.) Cr. Kita. - C. polyphylla W. K.) Schultr. Crnopac. - C. bulbifera (L.) Cr. Kita. 
Hutchinsia petraea (L.) Br. Vrelo i Zvonigrad u Zrmanji.

Capsella bursa pastoris (L.) Med. Vrelo u Zrmanji, Štikada, Ričice. Ljutoč. - C. rubella Rent. Vrelo u Zrmanji.

Draba verna L. Vrelo Rakovnik i Zvonigrad u Zrmanji; Crnopac (Lengyel).

Arabis turrita L. a) typica Beck. Trovrha, Zvonigrad. Ljutoč, Kita, Tremoina. - A. alpina L. Ćelavac, Kita. - A. hirsuta Scop. Ljutoč, V. Vrbica, Celavac, Crnopac, Kita. - var. exauriculata Borb. Ljutoč. - A. sagittata $D C$. Trovrha.

Erysimum erysimoides (L.) Fritsch. Štikada, Cerje, Gudura, Ljutoč.

Alyssum alyssoides L. Zvonigrad.

\section{Resedaceae.}

Reseda lutea L. Cerje, Ljutoč.

\section{Crassulaceae.}

Sedum roseum (L.) Scop. Crnopac, Bat. - S. maximum (L.) Hoffm. Trovrha, Ljutoč, Ćelavac, Kita, Tremzina. - S. hispanicum L., Crnopac. S. album L. Ploča, Trovrha, Ćelavac, Kita, Crnopac. - S. boloniense Lois. Štikada, Ričice, Cerje, Ljutoč, V. Vrbica, Ćelavac, Zagaljen, Vratce, Kita, Tremzina. S. ochroleucum Chaix. Trovrha, Vrelo u Zrmanji, V. Vrbica, Ćelavac, Crnopac, Kita, Tremzina. - S. altissimum Poir. Ploča (Kitaibel).

Sempervivum Schlehani Schott. V. Vrbica, Ćelavac, Crnopac, Kita, Tremzina.

\section{Saxifragaceae.}

Saxifraga aizoon Jacq. var. Malyi Schott. Trovrha, V. Vrcica, Celavac, Crnopac, Kita, Tremzina. - S. tridactylites $L$. Vrelo i Zvonigrad u Zrmanji. S. rotundifolia $L$. var. lasiophylla Schott. Trovrha, Ljutoč, Ćelavac, Crnopac, Kita, Tremzina.

Ribes grossularia L. Trovrha, V. Vrbica, Celavac, Crnopac, Kita. - R. alpinum L. var. palidigemmum Simk. V. Vrbica, Celavac, Crnopac, Kita, Tremzina.

\section{Rosaceae.}

Spiraea cana W. K. Trovrha, Crnopac, Kita, Ćelavac, Ljutoč. Na posljednja dva mjesta mnogobrojna. 
Aruncus silvester Kostel. Crnopac.

Cotoneaster integerrima Med. V. Vrbica, Ćelavac. -- var. parvifrons Borb. Crnopac. - C. tomentosa (Ait.) Lindl. Crnopac.

Pirus piraster (L.) Borkh. Vrelo u Zrmanji, Cerje, Ljuloč. Malus silvestris (L.) Mill. Vrelo u Zrmanji.

Sorbus aucuparia L. Ljutoč, Kita, Tremzina. - S. aria (L.) Cr. Trovrha, Ljutoč, V. Vrbica, Ćelavac, Kita. - var. lanifera Kern. Tremzina.

Amelanchier ovalis Med. Trovrha, V. Vrbica, Ćelavac, Kifa.

Crataegus monogyna Jacq. Vrelo u Zrmanji, Štikada, Cerje, Ljutoč, V. Vrbica, Ćelavac, Kita.

Rubus idaeus L. Trovrha, Ljutoč, V. Vrbica, Ćelavac, Crnopac, Kita, Tremzina. - R. saxatilis L. Crnopac (Zelebor, Rossi) Kita. - R. ulmifolius Schott. Vrelo u Zrmanji Šloser kao $R$. cinereus Rchb. a Vukotinović kao $R$, discolor Whe), Rakovnik. - R. tomentosus Borkh. Ploča (Kitaibel, Rossi). $-R$. hirtus W. K. Ljutoč, Rašlje, Kita. - R. caesius L. Gudura.

Fragaria vesca L. Vrelo u Zrmanji, Ljutoč, Tremzina. Fr. moschata Duch. Ljutoč, Ćelavac, Kita, Tremzina.

Potentilla erecta (L.) Hampe. Štikada, Ričica, Ljutoč, V. Vrbica, Crnopac, Tremzina. - P. reptans L. Ričica, Cerje, Ljutoč. - P. canescens Bess. Ćelavac. - P. pedata Nestl. Trovrha, Štikada, Ljutoč, Ćelavac, Crnopac. - P. australis Viraš. V. Vrbica, Zagaljen, Ljutoč. - P. micrantha Ram. Vrelo u Zrmanji, na podnožju Crnopca.

Geum urbanum L. Ljutoč, V. Vrbica, Ćelavac, Zagaljen, Tremzina.

Filipendula hexapetala (L.) Maxim. V. Vrbica, Tremzina.

Agrimonta eupatoria L. Trovrha, Gudura, Ričice, Gerje, Ljutoč, V. Vrbica, Vratce, Kita.

Aremonia agrimonioides (L.) Neck. Ljutoč, V. Vrbica, Crnopac.

Sanguisorba muricata (Spach) Gremli. Ljutoč, V. Vrbica, Vratce, Kita, Tremzina.

Rosa baldensis Kerner. Cerje, Gudura. - R. rubrifolia Vill. Ljutoč, Kita. - R. ferruginea Vill. Komić, Trovrha. $-R$. glaucescens Vulf. Trovrha, Crnopac. $-R$. pracrupticola Braun. Trovrha. Ona sa Kite jest: „Eine sich der $R$. rubrifolia var. praerupticola H. Braun. nähernde Form, doch ist die Behaarung 
weniger dicht und Stacheln mehr gekrümmt. Dr. Degen in sched. - R. canina L. Ričice. - R. fissidens Borb. Grnopac. - R. podolica Tratt. Crnopac. - R. syntrichostyla Rip. var. semibiserrata Borb. Grnopac. $-R$. biserrata Mórat. Rastovnik. - R. soltitialis Bess. Trovrha. - $R$, pendulina L. Grnopac, Kita, Tremzina. - R. gentilis Stern. V. Vrbica, Ćelavac, Tremzina. - var. adenoneura Borb. Crnopac. $-R$. spinosissima $L$. V. Vrbica, Kita. - var. megalacantha Borb. Ćelavac, Kita.

Prunus spinosa L. Štikada, Ričice, Ljutoč, Kita, Tremzina. - Pr. mahaleb L. V. Vrbica, Ćlavac, Kita.

\section{Leguminosae.}

Genista sagitalis L. Kita, Tremzina. - G. pubescens Láng. Trovrha. - G. dalmatica Bartl. var. dinarica Janchen. Trenzina. - G. silvestris Scop. V. Vrbica.

Laburnum alpinum (Mill.) Griseb. Trovrha.

Ononis spinosa L. Cerje, Ričice, Štikada, Gudura, Ljutoč, V. Vrbica, Vratce, Tremzina. - floribus albis. Ričice, Vratce. Medicago prostrata Jacq. Vratce, V. Vrbica. - M. falcata L. Štikada, Ričice, Cerje. - M. Lupulina L. Ričice, Ljutoč, V. Vrbica, Ćelavac, Vratce. Ljutoč.

Melilotus officinalis L. Vrelo u Zrmanji, Ričice, Cerje,

Trifolium rubens $L$. Trovrha, Ćelavac. $-T r$. alpestre $L$. V. Vrbica, Kita, Tremzina. - Tr. medium L. Tremzina. - Tr. pratense L. Ljutoč, Vratce, Kita. - var. pilosum Heuffel. Trovrha. - Tr. ochroleucum Huds. Trovrha, Ljutoč, Rašlje, Kita. - Tr. arvense $L$. Vratce, Štikada, Ljutoč. - Tr. fragiferum $L$. Ričice. - Tr. montanum L. Trovrha, V. Vrbica, Kita, Tremzina. - Tr. repens L. Štikada, Ričice, Cerje, Ljutoč, V. Vrbica, Vratce, Kita, Tremzina. - Tr. campestre Schreb. Trovrha, Ljutoč, Kita.

Anthyllis Jacquini Kerner. V. Vrbica, Ćelavac, Tremzina. A. Vulneraria L. Tremzina. - A. rubicunda Wender. Ćelavac. Dorycnium germanicum (Gremli) Rong. Trovrha, Ričice, Ljutoč, V. Vrbica, Čelavac, Kita, Tremzina.

Lotus corniculatus L. Komić, Cerje, Ljutoč, V. Vrbica, Vratce, Kita, Tremzina, - var. ciliatus Koch. Vratce, Ljutoč. var. hirsutus Koch Ljutoč. 
Colutea arborescens L. Vrelo u Zrmanji, (Klekovski, Rossi). Astragalus illyricus Bernh. Vrelo u Zrmanji.

Coronilla varia L. Ričice, Cerje, Ljutoč, V. Vrbica, Kita, Tremzina.

Vicia cracca L. Štikada, Ričice, Ljutoč, V. Vrbica. - V. lathyroides L. Zvonigrad. - V. sativa L. Štikada.

Lathyrus pratensis L. Ričice. - var. sepium Scop. Ljutoč, V. Vrbica, Tremzina. - L. megalanthus Stendel. Izmedju Ploče i Udbine (Poscharsky) V. Vrbica. - L. vernus (L.) Bernh. Na području Crnopca.

\section{Geraniaceae.}

Geranium columbinum L. Štikada. - G. molle L. Vrelo u Zrmanji. - G. rotundifolium L. Zvonigrad. - G. robertianum L. Trovrha, Ljutoč, Ćelavac, Vratce, Kita, Tremzina. - G. purpureum L. Rakovnik, Zagaljen. - G. macrorrhirum L. Trovrha, V. Vrbica, Čelavac, Crnopac, Kita. - G. sanguineum L. Trovrha, V. Vrbica, Ćelavac, Tremzina.

Erodium cicutarium (L.) L. 'Hérit. Vrelo u Zrmanji, Vratce, Gudura, V. Vrbica.

\section{Oxalidaceae.}

Oxalis acetosella L. Kita, Tremzina.

\section{Linaceae.}

Linum catharticum L. Trovrha, Ljutoč, V. Vrbica, Ćelavac, Vratce, Kita, Rašlje, Tremzina. - L. tenuifolium L. Trovrha. L. usitatissimum L. Vrelo a Zrmanji kult.

\section{Rutaceae.}

Ruta divaricata Ten. V. Vrbica, Ćelavae, Zagaljen, Crnopac, Kita.

\section{Polygalaceae.}

Polygala vulgaris L. var. albida Chodat. V. Vrbica.

\section{Euphorbiaccae.}

Mercurialis perennis L. V. Vrbica, Ćelavac, Kita, Trenzina. Euphorbia angulata Jacq. Kita. - E. verrucosa L. Tremzina. - E. helioscopia L. Vrelo u Zrmanji. - E. amygdaloides 
L. Ljutoč, Kita. - E. cyparissias L. Vrelo u Zrmanji, Štikada. - E. paralias L. Vrelo u Zrmanji (Klekovski). $-E$. exigua $L$. Ljutoč. - E. falcata L, Štikada, Ćelavac, Vratce.

\section{Anacardiaceae.}

Cotinus coggygria Scop. Trovrha, Kita.

\section{Celastraceae.}

Evonymus verrucosa Scop. Trovrha. - E. latifolia (L.) Mill. Trovrha. - E. vulgaris Mill. Ljutoč.

\section{Aceraceae.}

Acer monspessulanum L. Trovrha. - A. pseudoplatanus $L$. Trovrha, Cerje, Ljutoč, Crnopac, Kita, Tremzina. - A. obtusatum Kit. Trovrha, Palanka u Zrmanji, Štikada, Ljutoč, V. Vrbica, Celavac, Crnopac, Kita, Tremzina. - A. platanoides $L$. Trovrha, Kita. - A. campestre L. Trovrha, Štikada, Ričice, Cerje. - var. austriacum (Tratt.) DC. forma Bierbachii Schwerin. Komić na području Trovrhe.

\section{Rhamnaceae.}

Paliurus spina Christi Mill. Vrelo i Zvonigrad u Zrmanji. Rhamnus cathartica L. Ćelavac. - Rh. intermedia Steud. et Hochst. Rakovinik u Zrmanji. - Rh. fallax Boiss. Trovrha, Ljutoč, V. Vrbica, Crnopac, Kita. - Rh. saxatilis Jacq. V. Vrbica. - Rh. rupestris Scop. Trovrha, Kita. - Rh. frangula $L$. Ćelavac, Zagaljen.

\section{Vitaceae.}

Vitis vinifera $L$. Vrelo u Zrmanji, goji se na veliko. $-V$. silvestris Gmel. Štikada.

Tiliaceae.

Tilia platyphyllos Scop. Ričice, Cerje. - T. flava Wolny. Komić, Vrelo u Zrmanji; Crnopac (Janchen, Rossi).

Malvaceae.

Malva moschata L. Štikada, Kita. - var. laciniata Vis. Štikada, Ljutoč, Kita. - M. silvestris L. Štikada, Ričice, Cerje, Gudura, Ljutoč. 


\section{Guttiferae.}

Hypericum hirsutum L. Trovrha, Celavah, Kita, Tremzina. - H. perforatum L. Trovrha, Štikada, Ričice, Cerje, Ljutoč, V. Vrbica, Celavac, Vratce, Kita, Tremzina. - H. veronense Schrk. Ljutoč, Voda Vrbica. Zagaljen, Konopac. - H. montanum L. B. scabrum Koch. Trenzina. - H. alpigenum Kit. Trenzina.

\section{Cistaceae.}

Helianthemum hirsutum (Thnill.) Mérat forma obscurum (Pers.) Janchen. Trovrh, Ljutoč, V. Vrbica, Ćelavac, Vratce, Kita, Trem-zinn. - forma litorale (Willkomm) Janchen. V. Vrbica (Janchen), Crnopac (Degen, Rossi). - H. italicum (L.) Pers. Tremzina. H. alpestre (Jacq.) DC forma hirtum (Koch.) Pacher. V. Vrbica, Tremzina.

Fumana nudifolia (Lam.) Janchen. Kita.

\section{Violaceae.}

Viola arvensis Murr. a) ruralis Jord. Vrelo i Zvonigrad u Zrmanju. - V. tricolor L. Ćelavac. - V. odorata L. Vrelo u Zrmanji. - V. alba Bess. Vrelo i Zvonigrad. - V. silvestris Lam. Trovrha, Vrelo u Zrmanji, Ljutoč.

Thymelaeceae.

Daphne mesereum L. Trovrha, Kita. - D. alpina L. var. petiolaris Kessl. Trovrha, Zagaljen, Crnopac, Kita.

\section{Lytraceae.}

Lythrum salicaria L. Štikada, Cerje, Gudura.

\section{Oenotheraceae.}

Epilobium montanum L. Trovrha, Ljutoč, Ćelavac, Kita.

Chamaencrion angustifolium L. Ljutoč, Kitk.

Circaca lutetiana L. Ljutoč.

Araliaceae.

Hedera helix L. Vrelo i Zvonigrad u Zrmanji, Ljutoč, Kita, Tremzina. 
Umbelliferae.

Sanicula europaea L. Ljutoč, Kita, Tremzina.

Eryngium amethystinum L. Trovrha, Štikada, Gudura, Ričice, Cerje, Ljutoč, V. Vrbica, Vratce, Tremzina.

Chaerophyllum bulbosum L. Komić, Śtikada, Cerje.

Anthriscus fumarioides (W. K.) Spr. Trovrha, Celavac, Crnopac, Kita, Tremzina.

Scandix pecten Veneris L. Vrelo i Zvonigrad u Zrmanji.

Torilis anthriscus (L.) Gmel. Štikada, Ričice, Cerje, Ljutoč, V. Vrbica, Vratce.

Caucalis daucoides L. Vrelo u Zrmanji.

Orlaya grandiflora (L.) Hoffm. Vrelo u Zrmanji, Štikada, Ričice, Vratce, Kita.

Smyrnium perfoliatum L. Štikada, Vrelo u Zrmanji.

Physospermum verticillatum (W. K.) Vis. Ploča (Poscharsky), Trovrha, V. Vrbica, Crnopac, Kita.

Bupleurum Sibthorpianum Sm. Crnopac, Tremzina. - B. junceum L. Trovrha, Ljutoč, Ćelava. $-B$. aristatum Bartl. Vrelo i Rakovnik u Zrmanji, Štikada, Ljutoč, V. Vrbica, Vratce. var nanum Koch. Ploča (Poscharsky), Komić, Ljutoč, V. Vrbica, Vratce, Crnopac.

Apium repens (Jacq.) Rchb. Zrmanja (Schlosser).

Carum carvi L. Štikada, Tremzina.

Pimpinella major (L.) Huds. Ljutoč, Kita. - P. saxifraga $L$. Trovrha, Ričice, Ljutoč. - P. alpina Host. Crnopac.

Seseli elatum L. Trovrha.

Libanotis montana Cr. var. nitens Vis. Crnopac (Janchen, Rossi).

Athamanta Haynaldi Borb. et Mechtr. V. Vrbica, Ćelavac, Crnopac, Kita.

Cnidium silaifolium (Jacq,) Simk. Trovrha, Ljutoč, V. Vrbica, Ćelavac, Zagaljen, Crnopac, Tremzina.

Ligusticum dinaricum G. Beck. Kifa.

Ferulago zalbanifera Koch. Ploča (Poscharsky), Trovrha, Štikada.

Opopanax chironium (L. Koch. Štikada.

Peucedanum austriacum (Jacq.) Koch. var. montanum Koch. Trovrha, Ljutoč, Crnopac, Kita.

Pastinaca opaca Bern. Štikada. 
Heracleum sphondylium L. Tremzina, - H. ternatum Borb. Komić, Crnopac, Kita.

Laserpitium latifolium L. a) typicum Beck. Trovrha. - b) asperum (Cr) Beck. Trovrha. - L. siler L. V. Vrbica.

Daucus carota L. Ričice, Cerje, Ljutoč, V. Vrbica, Vratce.

Cornaceae.

Cornus mas L. Štikada, Ljutoč, Kita. - C. sanguinea $L$. Štikada, Cerje, Ljutoč, Celavac, Kita.

\section{Primulaceae.}

Primula vulgaris Huds. Vrelo i Zvonigrad, na podnožju Crnopca. - Pr. Columnae Ten. Trovrha, V. Vrbica, Ćelavac, Crnopac, Tremzina. Ričice.

Lysimachia punctata L. Ričice. - L. nummularia L. Gudura,

Anagallis arvensis L. Trovrha, Rakovnik, Gudura, Ljutoč, V. Vrbica, Kita, Tremzina.

Cyclamen europaeum L. Crnopac.

Oleaceae.

Fraxinus ornus L. Ploča (Beck.), Trovrha, Cerje, Ljutoč, Ćelavac, Kita.

\section{Gentianaceae.}

Centaurium minus Gars. Ljutoč, V. Vrbica.

Gentiana symphyandra Murb. Crnopac, Kita. - G. cruciata L. V. Vrbica, Crnopac. - G. tergestina Beck. Tremzina (Varga, Rossi). - G. utriculosa L. Tremzina.

Apocynaceae.

Vinca minor $L$. Zvonigrad.

Nerium oleander L. Vrelo u Zrmanji samoničenice (Klekovski). Ja toga nisam opazio, nego su mi kazivali, da ga zimi nemeću pod krov, nego ostane pod vedrim nebom.

Asclepiadaceae.

Cynanchum vincetoxicum (L.) Pers. Trovrha, Vrelo u $\mathrm{Zr}$ manji, Ljutoč, Celavac, Kita, Bat. 
Convolvulaceae.

Convolvulus arvensis L. Štikada, Ričice, Cerje, Gudura, Ljutoč, V. Vrbica, Vratce. - var. Cherleri (Ag.) Heldr. Štikada, Gudura, Vratce.

Calystegia sepium (L.) R. Br. Trovrha, Štikada.

Cuscuta europaea L. Ljutoč, Vrbica. - C. epithymum. (L.) Murr. Ljutoč, V. Vrbica, Ćelavac, Kita. Tremzina.

\section{Borraginaceae.}

Heliotropium europaeum L. var. gymnocarpum Borb. Vrelo u Zrmanji.

Cynoglossum montanum L. var. velebiticum Borb. Ljutoč, V. Vrbica, Celavac, Zagaljen, Kita, Tremzina.

Lappula echinata Gilib. Rakovnik.

Symphytum officinale L. Gudura. - S. Tuberosum L. Crnopac.

Pulmonaria officinalis $L$. Kita.

Myosotis scorpioides L. Štikada. - M. suaveolens $W . K$. Kita, Crnopac.

Lithospermum officinale L. Štikada, Ričice, Ljutoč, Kita. L. incrassatum Guss. Vrelo u Zrmanji.

Cerinthe minor $L$. Štikada, Ričice.

Echium vulgare L. Rakovnik, Štikada, Ljutoč, Vratce.

\section{Verbenaceae.}

Verbena officinalis L. Vrelo $\mathrm{u}$ Zrmanji, Gudura, Ričice.

\section{Labiatae.}

Ajuga reptans L. Kita. - A. genevensis L. Crnopac, Kita, Tremzina.

Teucrium Arduini L. Ćelavac, Zagaljen, Crnopac, Bat. $T$. chamaedrys $L$. var. illyricum Borb. Vrelo i Zvonigrad, Štikada, Zagaljen, Ćelavac, Kita, Tremzina. - T. montanum L. V. Vrbica, Ćelavac, Kita, Tremzina. - var. supinum Jacq. Trovrha, V. Vrbica, Celavac, Crnopac, Tremzina.

Scutellaria altissima L'. Ploča (Šloser, Vukotinović), Trovrha, V. Vrbica, Ćelavac, Crnopac (Janchen, Rossi).

Marrubium vulgare L. Štikada, Ričice, Vrbica, Vratce. M. candidissimum L. Komić, Rakovnich, Štikarda, Ričica, Gudura, 
Ljutoč, V. Vrbica, Zagaljen, Vratce.

Nepeta pannonica (L.) Jacq. Lomić, Štikarda, Vrbica, Ćelavac. - N. Cataria L. Rakovnik, Vratce, Štikada, Ljutoč, Crnopac.

Glechoma hederacca L. Vrelo i Zvonigrad. - Gl. hirsuta W. K. Vrelo u Zrmanji.

Brunella grandiflora (L,) Jacq. V. Vrbica. - Br. vulgaris $L$. Ljutoč, Kita. - Tremzina. - var. pinnatifida Koch. Kita. Br. alba Pallas. Ričice, Cerje, Gudura, Ljutoč, V. Vrbica, Vratce, Tremzima. - var. pinnatifida Koch. Ljutoč, V. Vrbica, Vratce, Kita, Tremzina.

Melittis albida Guss. Kita.

Galeopsis ladanum L. Śtikada. - G. canescans Bess. Rakovnik. - G. pusbescees. Ćelavac, Crnopac.

Lamium luteum (Huds.) Kroch. Ljutoč. - L. amplexicaule L. Zvonigrad. - L. purpereum L. VreJo i Zvonigrad. - L. bifidum Cyr. Dosele samo kod Vrela u Zrmanji. - L. laculatum $L$. Vrelo i Zvonigrad, Ričice, Ćelavac, V. Vrbica. - L. album $h$. Vrelo u Zrmanji.

Leonurus cardiaca L. Štikada, Gudura.

Ballotta nigra L. Vratce, Gudura.

Stachys italica Mill. U Zrmanji (Šloser, Vukotinović, Klekovski) - St. germanica L. Štikada, Gudura, Ričice, Ljutoč, V. Vrbica, Ćelavac, Zagaljen, Vratce, Crnopac. - St. silvatica L. Gudura. - St. palustris L. Ričice, Gudura, Štikada. - St. annua L. Gudura, Lomić. - St. suberenata Vis. Tremzina. var. angustifolia Vis. V. Vrbica, Ćelavac, Tremzina. - var. sarajevensis Maly. Crnopac. - St. recta L. Štikada, Ričice, Cerje. - St. obliqua W. K. Izmedju Udbine i Ploča (Poscharsky), Vrelo u Vrmaji (Klekovski), Vratce, Stikada. - St. serotina (Host) Fritsch Vratce, Celavac. - var. Dalmatica Degen. Vrelo u zrmanji, Tremzina. - St. velebitica (Kerner) Eritsch. var hirticalyx Degen. Trovrha.

Salvia verticillata L. Trovrha, Gudura, Ričice, Cerje, Ljutoć, V. Vrbica. - S. glutinosa L. Gudura, Ljutoč, Ćelavac, Lita. S. officinalis L. Vrelo u Zrmanji. Kazivali su mi, da je ima kod Ričice blizu kućah Tomičićevih. - S. pratensis L. Gudura, Ričice, Ljutoč, V. Vrbica, Vratce, Tremzina.

Melissa officinalis. Vrelo u Zrmanji. 
Satureia subspicata Vis. Crnopac (Zelebor), V. Vrbica, Tremzina. - S. variegata Host Vrelo i Zvonigrad, Ljutoč, V. Vrbica, Vagaljen, Crnopac, Tremzina. - S. vulgaris (L.) Fritsh. Trovrha, Ričice, Kjutoč, V. Vrbica, Ćelavac, Vratce, Crnopac, Kita, Tremzina. - S. acinos (L.) Scheele. Trovrha, Ričice, Ljutoč; Crnopac, (Zelebor, Rossi). - S. thymifolia Scop. Ploča, (Flora croat.) Trovrha, Ljutoč, V. Vrbica, Celavc, Zagaljen, Vratce, Crnopac, Bat, Rašlje, Tremzina. - S. calamintha (L.) Scheele var. intermedia Baumg. Trovrha, Ljutoč, V. Vrbica, Ćelavac, Kita, Rašlje.

Origanum vulgare L. var. latebracteatum Beck. Trovrha, Štikarda, Ričice, Ljutoć, V. Vrbiba, Ćelavac.

Thymus acicularis W. K. forma subalpinus Degen. Crnopac. - Th. subcitratus Schreb. Zrmanja (Hacquet kao Th. Serpyllum) Ploča, Cerje, Ljutoč, V. Vrbica, Ćelavac, Vratce, Kita, Tremzina.

Lycopus europaeus L. Štikada, Ričica.

Mentha candicans Ex. var stenanthelmia Borb. et Waisb. Ljutoč, V. Vrbica. - M. spicata L. Štikada, Ričice, Cerje, Ljutoč, V. Vrbica, Vratce. - M. cuspidata Opiz. Crnopac. - M. pulegium L. Štikada, Gudura, Ričice.

\section{Solanaceae.}

Hyosciamus niger L. Štikada.

Solanum dulcamara L. Štikada, Ričice, Ljutoč, Ćelavac, Kita. - S. nigrum L. Vrelo u Zrmanji, Štikada, Ričice, Cerje, Celavac.

Datura Stramonium L. Štikada.

Scrophulariaceae.

Verbascum blattaria L. Ričice. - V. thapsiforme L. Štikada. V. phlomoides L. Ljutoč, V. Vrbica, Ćelavac, Vratce, Kita. $V$. floccosum W. K. Gudura. - V. Fluminense Kerner. Ploča, sa bijelim cvijetom. - V. Chaixii Vill. Ričice, Cerje, Crnopac. - V. nigrum L. Štikada, Gudura, Ljutoč, V. Vrbica, Ćelavac, Zagaljen, Vratce, Kita, Tremzina.

Cymbalalaria muralis G. M. Sch. Ljutoč, Ćelavac, Crnopac, Kikxia elatine (L.) Dum. Vratce.

Linaria vulgaris Mill. Komić, Štikada, Ričice, Cerje, Ljutoč, Vratce. - L. italica Trev. Vrelo i Zvonigrad u Zrmanji. 
Chaenorrhinum minus (L.) Lge. Trovrha, Rakovnik.

Scrophularia nodosa L. Trovrha, Ljutoč, Kita. - Scr. laciniata W. K. Ljutoč, V. Vrbica, Ćelavac, Zagaljen, Kita, Tremzina. - var. Pantocsekii Grisel. Trovrha, Crnopac. - Scr. canina L. Vrelo u Zrmanji, Štikada, Ričice, Vratce.

Gratiola officinalis L. Štikada, Ričice.

Veronica beccabunga L. Štikada, Gudura. - V. chamaedrys L. Štikada, Ričice. - V. officinalis L. Ljutoč, Ćelavac, Kita, Tremzina. - V. Jacquini Baumg. Štikada, Ljutoč, V. Vrbica. - V. spicata L. Trovrha, V. Vrbica, Celavac, Kita, Tremzina. - V. serpyllifolia L. Ljutoč, V. Vrbica, Kita, Tremzina. - V. Tournefortii Gmel. Vrelo u Zrmanji. - V. agrestis L. Gudura, V. Vrbica. - V. hederifolia L. Vrelo i Zvonigrad u Zrmanji.

Digitalis ambigua Murr. Kita, Tremzina. - D. Ferruginea L. Ploča (Kitaibel, Poscharsky, Rossi), Ljutoč. - D. laevigata $W . K$. Trovrha, Ljutoč.

Melampyrum arvense L. Štikada. -- M. vulgatum Pers. Trovrha, Ljutoč. - M. nemorosum L. Ćelavac.

Euphrasia Rostkoviana Hayne. Štikada, Gudura, Ljutoč. E. tatarica Fisch. Komić, Trovrha, Štikada, Ljutoč. - E. liburnica Wettst. Crnopac, Tremzina. - E. illyrica Wettst. V. Vrbica, Ćelavac. Nije tipička forma, već izmedju ove i $E$. salisburgensis var. coelurans.

Alectorolophus crista galli (L.) M. B. Gudura, Ričice. A. major (Ehrh.) Rchb. Sa prijašnjim.

\section{Orobanchaceae.}

Orobanche alba Steph. Ljutoč, Ćelavac, Tremzina.

\section{Globulariaceae.}

Globularia Willkommii Nym. V. Vrbica. - Gl. cordifolia L. Crnopac. - Gl. bellidifolia Ten. Kita, Tremzina.

\section{Plantaginaceae.}

Plantago media L. Ričice, Ljutoč, V. Vrbica, Vratce, Kita, Tremzina. - Pl. major L. Štikada, Ljutoč, Vratce, Tremzina. Pl. lanceolata L. Ričice, Ljutoč, Vratce, Tremzina, Kita, V. Vrbica, 
Štikada, Gudura. - var. capitellata Koch. Ljutoč, Tremzina. Pl. carniata Schrad. Trovrha, Komić, Gudura, Vratce, Kita, Tremzina.

\section{Rubiaceae.}

Sherardia arvensis L. Vrelo i Zvonigrad u Zrmanji.

Asperula odorata L. Trovrha, V. Vrbica, Celavac, Kita. A. canescens Vis. var. glabra Deg. Vrelo u Zrmanji. - A. longiflora W., K. Gudura, Ričice, Cerje, Ljutoč, V. Vrbica, Ćelavac, Zagaljen, Vratce, Crnopac, Kita, Tremzina.

Galium cruciata (L.) Scop. Zvonigrad, Cerje. - G. vernum Scop. Tremzina. - G. aparine L. Štikada, Gudura. - G. silvaticum $L$. Ćelavac. - G. verum L. Zvonigrad, Štikada, Gudura, Ričice, Cerje, Ljutoč, V. Vrbica, Vratce, Tremzina. - G. purpureum L. Trovrha, Komić, Ljutoč, V. Vrbica, Ćelavac, Zagaljen, Crnopac, Kita, Tremzina. - G. lucidum All. Komić, Trovrha, Ljutoč, V. Vrbica, Crnopac, Kita, Tremzina. - G. erectum Huds. V. Vrbica, Kita, Tremzina. - G. mollugo L. Komić, Štikada, Ričice, Cerje, Ljutoč, V. Vrbica, Ćelavac, Vratce, Kita, Tremzina.

\section{Caprifoliaceae.}

Sambucus nigra L. Ljutoč, Ćelavac. - S. ebulus L. Ljutoč, V. Vrbica, Ćelavac, Zagaljen, Vratce.

Viburnum lantana L. Ljutoč, V. Vrbica, Ćelavac, Zagaljen.

Lonicera xylosteum L. Trovrha, Ljutoč, Crnopac, Kita. L. alpigena L. Trovrha, Ljutoč, Ćelavac, Crnopac, Kita, Tremzina.

\section{Valerianaceae.}

Valeriana officinalis $L$. Ćelapac, Tremzina. - V. angustifolia Tausch. Trovrha, Ćelavac, Tremzina. - V. tripteris var. velebitica Deg. Ljutoč, V. Vrbica, Ćelavac, Kita, Tremzina.

\section{Dipsacaceae.}

Cephalaria lencantha (L.) Schrad. Zvonigrad.

Dipsacus silvestris Hunds. Štikada, Ričice, Cerje, Gudura, Ljutoč, Vratce. - D. laciniatus $L$. Ričice.

Knautia purpurea (Vill.) Borb. var. illyrica (Beck) Szabó. V. Vrbica, Ćelavac, Tremzina. - Var. dissecta Borb. V. Vrbica, Tremzina. - Kn. travnicensis Beck. Tremzina. - Var. Crassifolia Szabó. V. Vrbica (Janchen i Watzel). 
Scabiosa agrestis W.K. Komić, Trovrha, Ričice, Cerje, Ljutoč, V. Vrbica, Ćelavac, Vratce, Tremzina. - Sc. graminifolia W. K. Crnopac (Zelebor, Rossi) Kita.

\section{Campanulaceae.}

Campanula pyramidalis L. Ploča (Kitaibel, Rossi), Trovrha, Rakvonik, Ljutoč. - C. linifolia Scop. var. pinifolia Mechtr. Crnopac, Tremzina. - C. Scheuchzeri Vill. V. Vrbica, Kita, Tremzina. - C. Waldsteiniana R. et Sch. Crnopac (Zelebor, Rossi), Ploča, Trovrha, V. Vrbica, Ljutoč, Ćelavac, Zagaljen, Kita. - C. patula L. Štikada, Gudura. - C. rapunculus $L$. Vratce, Štikada, Ričice. - C. persicifolia L. Gudura, V. Vrbica, Trenzina. - C. trachelium L. Stikada, Ljutoč, Kita, Tremzina. C. bononiensis L. Vrelo u Zrmanji, Ljutoč. - C. glomerata L. var. elliptica Kit. V. Vrbica, Ćelavac, Kita, Tremzina.

Phyteuma orbiculare L. ssp. flexurorum R. Schltz. Ljutoč, Crnopac, Bat, Kita. - Ph. spicatum L. Rašlje na Crnopcu.

Edraianthus tennifolius (W.K.) A. D. C. Crnopac, Tremzina. - E. graminifolius (L) DC. Crnopac, Kita.

\section{Compositae.}

Eupatorium cannabinum L. Ljutoč, Kita.

Solidago alpestris W. K. V. Vrbica, Celavac, Kita.

Bellis perennis L. Vrelo i Zvonigrad, Štikada, Gudura, Ričice, Cerje.

Erigeron canadensis L. Štikada, Ričice. - E. acer L. Gudura, Ljutoč, Vratce, Kita. - E. polymorphus Baumg. Trovrha.

Filago arvensis $L$. Štikada, Gudura. - F. minima $L$. Štikada, Gudura.

Leontopodium alpinum L. Crnopac (Zelebor, Janchen).

Gnaphalium uliginosum L. Štikada, Gudura. - G. silvaticum L. Celavac, Crnopac, Tremzina.

Helichrysum italicum (Roth) Guss. Zvonigrad.

Inula ensifolia L. V. Vrbica, Celavac, Tremzina. - I. spiraeifolia $L$. Ljutoč. - I. conyza $D C$. Ljutoč. - I. britannica $L$. Štikada, Gudura, Ričice, Cerje. - I. hirta L. Ćelavac, Kita, Tremzina. -- I. oculus Christi L. Vrelo u Zrmanji (Klekovski, Rossi), Ploča, Štikada, Ljutoč, V. Vrbica. Vratce, Crnopac, Kita. 
Pulicaria vulgaris Gaertn. Štikada, Ričice. - P. disenterica (L.) Gray. Štikada, Gudura.

Buphthalmum salicifolium L. Ljutoč, V. Vrbica, Vratce, Kita. Xanthium spinosum $L$. Vratce, Štikada.

Bidens tripartitus L. Štikada, Ričice.

Anthemis arvensis L. Ričice, Cerje, Ćelavac.

Achillea clavenae L. Crnopac, Kita. - A. virescens (Fenzl.) Heimerl., Štikada, Ričice. Cerje, Ljutoč, V. Vrbica, Ćelavac, Zagaljen, Vratce, Crnopac, Kita, Tremzina. - A. millejolium $L$. Ričice, Cerje, Tremzina.

Matricaria chamomilla L. Štikada, Ričice.

Chrysanthemum leucanthemum L. Ričice, Cerje, Ljutoč, V. Vrbica, Vratec. - Ch. corymbosum L. Trovrha, V. Vrbica, Crnopac, Tremzina.

Artemisia absinthium L. Vratce, Štikada. - A. Lobelii All. V. Vrbica, Ćelavac.

Tussilago farfara L. Vrelo u Zrmanji, Vratce, Ričice, Ljutoč.

Petasites albus (L.) Gaertn. Tremzina.

Senecio vulgaris L. Štikada, Ričice, Cerje. - S. rupestris W. K. Trovrha, Ljutoč, V. Vrbica, Ćelavac, Zagaljen, Crnopac, Kita. - S. jacobaea L. Stikada, Ričice. - S. nemorensis $L$. Ljutoč. - S. Fuchsii Gmel. Trovrha, Ćelavac, Kita, Bat. - S. cacaliaster Lam. Crnopac (Janchen, Rossi). - S. doronicum $L$. Crnopac, Tremzina.

Carlina aggregata Willd. V. Vrbica, Zagaljen, Vratce, Tremzina. - C. vulgaris L. Ljutoč, V. Vrbica, Zagaljen, Vratce, Kita. Arctium tomentosum Mill. Gudura, Ljutoč. - A. lappa L. Štikada.

Carduus candicans W. K. Rašlje na Crnopcu. - C. carduelis (L.) Gren. var. alpestris W. K. Crnopac. - C. acanthoides L. Ričice, Cerje, Ljutoč, V. Vrbica, Ćelavac, Vratce.

Cirsium erisithales (L.) Scop. Kita. - C. Acarna (L.) Mnch. Vrelo u Zrmanji (Vukotinović, Klekovski, Rossi), Zvonigrad. C. lanceolatum (L.) Scop. Ričice, Cerje, Ljutoč, V. Vrbica, Zagaljen, Vratce, Rašlje. - C. eriophorum (L.) Scop. Ljutoč, Ćejavac, Zagaljen, Kita. - C. pannonicum (L. fil.) Gaud. V. Vrbica, Tremzina. - C. arvense (L.) Scop. Stikada, Ričice. var. argenteum Vest. Vrelo u Zrmanji, Stikada, Ričice. - $C$. 
acaule (L.) Web. Štikada, Ljutoč, V. Vrbica, Ćeiavac, Vratce, Rašlje, Kita, Tremzina.

Onopordum acanthium L. Štikada, Ričice.

Centuurea alba L. ssp. concolor DC. Ćelavac. - ssp. deusta Ten. Trovrha. - C. Haynaldi Borb. V. Vrbica, Tremzina. C. bracteata Scop. Ljutoč. - C. pannonica (Henff.) Hayek. Vratce, Štikada, Ričice. - C. variegata Lam. V. Vrbica, Tremzina. - var. adscendens Bartt. Crnopac. - C. cristata Bartt. Vrelo u Zrmanji. - C. cyamus L. Štikada, Cerje. - C. scabiosa L. Štikada, Gudura, Ričice, Cerje. - C. Fritschii Hayek var. spinigera Hayck. Vrelo u Zrmanji.

Scolymus hispanicus L. Vrelo u Zrmanji, Vratce.

Cichorium intybus L. Vratce, Ljutoč.

Lapsana communis L. Ljutoč, V. Vrbica.

Aposeris foetida (L) Less. Kita.

Hypochoeris radicata L. Štikada, Cerje. - H. illyrica Maly. V. Vrbica, Tremzina.

Leontodon autumnalis L. Vratce, Ričice. - L. danubialis Jacq. V. Vrbica, Zagaljen, Kita. - L. crispus Vill. V. Vrbica, Celavac, Tremzina. - L. Rossianus Degen et Lengyel. Crnopac, Bat.

Picris hieracioides L. Ṡtikada, Cerje. - P. spinulosa Bert. Vrelo u Zrmanji, Ljutoč, Vratce, Kita.

Tragopogon Tommasinii Schullz. Ljutoč, Voda, Vrbica, Vratce.

Scorzonera villosa Scop. Ričice, Cerje, Ćelavac.

Lagoseris sancta (L.) Maly. Rakovnik.

Taraxacum officinale Web. Vrelo u Zrmanji.

Sonchus laevis (L.) Gars. Ričice.

Lactuca perennis L. Trovrha, Crnopac. - L. vinnicea (L.) Presl. Ljutoč. - L. muralis (L.) Fres. Ljutoč, V. Vrbica, Ćelavac, Vratce, Crnopac, Kita, Tremzina. - L. salignza L. Ljutoč, Ćelavac.

Crepis foetida L. Vrelo u Zrmanji. - Cr. setosa Hall. fil. Štikada, Ričice, Cerje, Kita. - Cr. biennis L. Ričice, Cerje, Ljutoč, Vratce.

Prenanthes purpurea L. Trovrha, Ćelavac, Kita. 
Hieracium Hoppeanum Schult. ssp. leucocephalum Vukot. Ljutoč V. Vrbica, Vratce, Kita, Tremzina. - ssp. multisetum $N . P$. a) genuinum $\beta$ ) polyadenium $N . P$. Trovrha. - ssp. testi. moniale $N$. P. Kita. - H. pilosella L. ssp. minuticeps $N$. P. Ljutoč. - H. cymosum $W$. K. ssp. viridans $N$. P. V. Vrbica, Tremzina. - H. Bauhini Schult. ssp. marginale N. P. Ljutoč. - ssp. substoloniferum N. P. Crnopac (Degen), Vratce, V. Vrbica. - H. bupleuroides Gmel. ssp. Schenkii Griseb. Crnopac (Degen). - H. macrodon N. P. Ćelavac. - H. villosum ssp. villosum N. P. forma involucratum Rochel. Crnopac (Degen, Rossi). - H. scorzonerifolium Vill. ssp. flexuosum W. K. Trovrha, Kita, Bat. - var. caloescens Deg. et Zahn. Crnopac, Kita (Degen). - H. silvaticum L. ssp. bifidiforme Zahn. Ljutoč, Kita. - ssp. gentile Jord. Crnopac (Degen). - ssp. oblongum Jord. Ljutoč. - ssp. pleiophyllogenes Zahn. Crnopac (Degen). H. bifidum Kit, ssp. bifidum Kit. Crnopac (Degen, Rossi). ssp. Scandinaviorum Zahn. Tremzina. - H. Wiesbaurianum Mechtz. Tremzina. - H. Waldsteinii Tausch. ssp. lanifolium N. P. Crnopac (Degen), Trovrha. - H. humile Jacq. ssp. sarajevoënse $G$. Beck. Crnopac, Kita (Degen, Rossi). - H. integrifolium Lange a) genuinum Zahn. Trovrha. $-H$. Arpadianum Zahn. Trovrha. 



